\title{
Almost Periodic Solution for an Epidemic Prey-Predator System with Impulsive Effects and Multiple Delays
}

\author{
Baodan Tian, Ning Chen, and Yanhong Qiu \\ School of Science, Southwest University of Science and Technology, Mianyang 621010, China \\ Correspondence should be addressed to Baodan Tian; tianbaodan@swust.edu.cn
}

Received 28 March 2015; Accepted 16 August 2015

Academic Editor: Mauro Sodini

Copyright (C) 2015 Baodan Tian et al. This is an open access article distributed under the Creative Commons Attribution License, which permits unrestricted use, distribution, and reproduction in any medium, provided the original work is properly cited.

\begin{abstract}
A nonautonomous epidemic prey-predator system with impulsive effects and multiple delays is considered; further, there is an epidemic disease in the predator. By the mean-value theorem of multiple variables, integral inequalities, differential inequalities, and other mathematical analysis skills, sufficient conditions which guarantee the permanence of the system are obtained. Furthermore, by constructing a series of Lyapunov functionals it is proved that there exists a unique uniformly asymptotically stable almost periodic solution of the system.
\end{abstract}

\section{Introduction}

It is reported that there were more than twenty kinds of newly emerging viruses in the recent 30 years, such as Ebola, Hantavirus, AIDS, SARS, H5N1, and H7N9. Authoritative experts express the fact that the fundamental cause of these epidemic viruses is the loss of ecological balance and environmental degradation, and the basic rule between human and the nature is destroyed, which lead to the revenge of the nature and the invasion of the virus. Therefore, human, environment, and the disease are mutually affective and mutually restrictive.

Ecological epidemiology is a new subject to study the distribution and propagation rules between humans and other species; it is the combination of ecology and epidemiology, starting from the changes of ecological environment. And one of the most important aims of the ecological epidemiology is to study the propagation trend of the disease in the ecological systems so that the government can give corresponding controlling strategies in first time. Thus, it is very meaningful and valuable to study the dynamics of an epidemic ecological system. And in the recent years, more and more ecologists, epidemiologists, and mathematicians have devoted themselves to the study of the epidemic ecological models (see $[1-20]$ ), and most of the models are ordinary differential equations (see [1-15]) or functional differential equations (see [16-20]), respectively. For example, Xiao and Bosch [1] derived the following ecoepidemic model with disease in the predator in 2003:

$$
\begin{aligned}
& \frac{d x}{d t}=x\left(r_{1}-a x-b_{1} y_{1}-b_{2} y_{2}\right), \\
& \frac{d y_{1}}{d t}=y_{1}\left(r_{2}+c x-d_{1}\left(y_{1}+y_{2}\right)-\beta y_{2}\right), \\
& \frac{d y_{2}}{d t}=y_{2}\left(\beta y_{1}-d_{2}\left(y_{1}+y_{2}\right)\right),
\end{aligned}
$$

in which mathematical analyses of the model equations with regard to invariance of nonnegativity, boundedness of solutions, nature of equilibria, permanence, and global stability are analyzed.

The environment varies due to the factors such as seasonal effects of weather, food supplies, mating habits, and harvesting. So it is more reasonable to assume the periodicity or almost periodicity of parameters in the systems. And considering this factor, Tian et al. [21] considered the following nonautonomous epidemic prey-predator system in 2009:

$$
\begin{aligned}
& X^{\prime}(t) \\
& =X(t)\left[r_{1}(t)-a(t) X(t)-b_{1}(t) S(t)-b_{2}(t) I(t)\right],
\end{aligned}
$$




$$
\begin{aligned}
& S^{\prime}(t) \\
& \quad=c(t) X(t-\tau) S(t-\tau) \\
& \quad+S(t)\left[-r_{2}(t)-d_{1}(t)(S(t)+I(t))-e(t) I(t)\right], \\
& \quad I^{\prime}(t)=I(t)\left[e(t) S(t)-d_{2}(t)(S(t)+I(t))\right] .
\end{aligned}
$$

On the other hand, it is known that the ecoepidemic system will often be perturbed by the factors of human exploitation activities, such as planting and harvesting. Among these human disturbances, periodic or almost periodic and instantaneous perturbations are the most common, and these perturbations can be regarded as the impulsive effects when modeling the system. If the factors of time delays are also considered, then the corresponding models should be described as impulsive functional differential equations. However, to the best our knowledge, references on the periodic ecoepidemic model with impulsive perturbations seem relatively fewer. Since the rapid development in the theory of the impulsive equations and the functional differential equations (see [21-26]), many excellent results have been derived in ecological models, epidemic models, and even neural network models (see [27-37] and so on).

Enlightened by the above work, in this paper we will propose an epidemic ecological prey-predator model with impulsive perturbations and three kinds of time delays. First, we generalized the term $a(t) X(t)$ into $a(t) X\left(t-\tau_{1}\right)\left(\tau_{1}>0\right)$ in the present model, which means the negative feedback intermediate prey crowding. Second, we consider there is a time $\tau_{2}>0$ of digestion of the susceptible predator. Third, we consider there is a latency period $\tau_{3}>0$ before an infected predator could infect a susceptible predator. The final model we will study in this paper is as follows:

$$
\begin{aligned}
& X^{\prime}(t)=X(t) \\
& \cdot\left[r_{1}(t)-a(t) X\left(t-\tau_{1}\right)-b_{1}(t) S(t)-b_{2}(t) I(t)\right] \\
& S^{\prime}(t)=c(t) X\left(t-\tau_{2}\right) S\left(t-\tau_{2}\right)+S(t) \\
& \quad \cdot\left[-r_{2}(t)-d_{1}(t)(S(t)+I(t))-e(t) I\left(t-\tau_{3}\right)\right] \\
& I^{\prime}(t)=e(t) S(t) I\left(t-\tau_{3}\right)-d_{2}(t)(S(t)+I(t)) I(t), \\
& X\left(t_{k}^{+}\right)=\left(1+h_{1 k}\right) X\left(t_{k}\right) \\
& S\left(t_{k}^{+}\right)=\left(1+h_{2 k}\right) S\left(t_{k}\right) \\
& I\left(t_{k}^{+}\right)=\left(1+h_{3 k}\right) I\left(t_{k}\right),
\end{aligned}
$$

where $X(t)$ denotes the density of the prey, $S(t)$ and $I(t)$ denote the density of the susceptible and the infected predators, $r_{i}(t)(i=1,2)$ means the intrinsic rate of natural increase, and the minus before $r_{2}(t)$ means that the susceptible predator is dependent on the prey. $a(t)$ denotes the coefficient of the density dependence of the prey, $d_{i}(t)(i=1,2)$ denotes the competitive coefficients between the predators, $b_{i}(t)(i=1,2)$ means the preying capacity of the susceptible and the infected predators, $c(t)$ means the relative preying capacity of the susceptible predator, and $e(t)$ means the touching rate between the susceptible predators.

$h_{i k}>-1$ denotes the impulsive effects. When $h_{i k}>0$, the effects represent planting, when $h_{i k}<0$, the effects denote harvesting.

Throughout the present paper, we define

$$
\begin{aligned}
f^{u} & =\sup _{t \in R^{+}} f(t), \\
f^{l} & =\inf _{t \in R^{+}} f(t),
\end{aligned}
$$

for any bounded function $f(t)$ defined on $R^{+}=[0,+\infty)$.

Further, we assume that

(C1) $a(t), c(t), e(t), b_{i}(t)$, and $d_{i}(t)(i=1,2)$ are all bounded and positive almost periodic functions;

(C2) $H_{i}(t)=\prod_{0<t_{k}<t}\left(1+h_{i k}\right)$ is almost periodic functions and there exist positive constants $H_{i}^{u}$ and $H_{i}^{l}$ such that $H_{i}^{l} \leq H_{i}(t) \leq H_{i}^{u}, i=1,2,3$.

The rest of this paper is organized as follows. In Section 2, we will give several useful lemmas for the proof of our main results. In Section 3, we will state and prove our main results such as the permanence of the system and the existence and the uniqueness of almost periodic solution which is uniformly asymptotically stable by constructing a series of Lyapunov functional. In the last section, we will give some discussions and give a brief summary for the paper.

\section{Preliminaries}

$K=\left\{t_{k} \in R \mid t_{k}<t_{k+1}, k \in N, \lim _{k \rightarrow \pm \infty} t_{k}= \pm \infty\right\}$, and we denote the set of all sequences that are unbounded and increasing. Let $D \subset \Omega, \Omega \neq \Phi, \tau=\max _{1 \leq i \leq 3}\left\{2 \tau_{i}\right\}$, $\xi_{0} \in R$. Also, we denote $P C\left(\xi_{0}\right)$ as the space of all functions $\phi:\left[\xi_{0}-\tau, \xi_{0}\right] \rightarrow \Omega$ having points of discontinuity at $\mu_{1}, \mu_{2}, \ldots, \mu_{n}, \ldots \in\left[\xi_{0}-\tau, \xi_{0}\right]$ of the first kind, being left continuous at these points.

For $J \subset R, P C(J, R)$ is the space of all piecewise continuous functions from $J$ to $R$ with points of discontinuity of the first kind $t_{k}$, at which it is left continuous.

Let $\phi_{1}, \phi_{2}, \phi_{3} \in P C(0)$, denote $X(t)=X\left(t ; 0, \phi_{1}\right), S(t)=$ $S\left(t ; 0, \phi_{2}\right), I(t)=I\left(t ; 0, \phi_{3}\right)$, and $X, S, I \in \Omega$ is the solution of system (3) satisfying the following initial conditions:

$$
\begin{aligned}
& 0 \leq X\left(s ; 0, \phi_{1}\right)=\phi_{1}(s)<\infty, \\
& 0 \leq S\left(s ; 0, \phi_{2}\right)=\phi_{2}(s)<\infty, \\
& 0 \leq I\left(s ; 0, \phi_{3}\right)=\phi_{3}(s)<\infty,
\end{aligned}
$$

$$
s \in[-\tau, 0]
$$

$$
\begin{aligned}
& X\left(0 ; 0, \phi_{1}\right)=\phi_{1}(0)>0, \\
& S\left(0 ; 0, \phi_{2}\right)=\phi_{2}(0)>0, \\
& I\left(0 ; 0, \phi_{3}\right)=\phi_{3}(0)>0 .
\end{aligned}
$$


Lemma 1 (see [38]). (1) Assume that, for $x(t)>0, t \geq 0$, the following holds:

$$
x^{\prime}(t) \leq x(t)(a-b x(t-\tau))
$$

with initial conditions $x(s)=\phi(s) \geq 0, s \in[-\tau, 0]$, where $a$, $b$ are positive constants; then there exists a positive constant $x^{*}$ such that

$$
\lim _{t \rightarrow \infty} \sup x(t) \leq x^{*}:=\frac{a e^{a \tau}}{b} .
$$

(2) Assume that, for $x(t)>0, t \geq 0$, the following holds:

$$
x^{\prime}(t) \geq x(t)(c-d x(t-\tau))
$$

with initial conditions $x(s)=\phi(s) \geq 0, s \in[-\tau, 0]$, where $c$ and $d$ are positive constants; then there exists a positive constant $x_{*}$ such that

$$
\lim _{t \rightarrow \infty} \inf x(t) \geq x_{*}:=\frac{c e^{\left(c-d x^{*}\right) \tau}}{d} .
$$

Also, as a direct application of the conclusion in the proof of Corollary 4.1 in [39], we can obtain the following lemma.

Lemma 2. For $a>b>c>0, x(0)>0$.

(1) If $x^{\prime}(t) \geq a x(t-\tau)-b x(t)-c x^{2}(t)$, then for any $\varepsilon>0$, there exists a constant $T_{1}>0$, such that

$$
x(t) \geq \frac{a-b}{c}-\varepsilon, \quad \text { when } t \geq T_{1}+\varepsilon .
$$

That is,

$$
\lim _{t \rightarrow \infty} \inf x(t)>\frac{a-b}{c} .
$$

(2) If $x^{\prime}(t) \leq a x(t-\tau)-b x(t)-c x^{2}(t)$, then for any $\varepsilon>0$, there exists a constant $T_{2}>0$, such that

$$
x(t) \leq \frac{a-b}{c}-\varepsilon, \quad \text { when } t \geq T_{2}+\varepsilon .
$$

That is,

$$
\lim _{t \rightarrow \infty} \sup x(t)<\frac{a-b}{c} .
$$
delay:

If we consider the following almost periodic system with

$$
x^{\prime}(t)=f\left(t, x_{t}\right), \quad t \in R,
$$

and the associate product system of system (14) is in the form of

$$
\begin{aligned}
& x^{\prime}(t)=f\left(t, x_{t}\right), \\
& y^{\prime}(t)=f\left(t, y_{t}\right),
\end{aligned}
$$

$$
t \in R
$$

then, by the conclusions of [23], one has the following lemma.
Lemma 3 (see [23]). For $\phi, \psi \in C_{B}$, suppose that there exists a Lyapunov function $V(t, \phi, \psi)$ defined on $R^{+} \times C_{B} \times C_{B}$ satisfying the following three conditions:

(1) $u(\|\phi-\psi\|) \leq V(t, \phi, \psi) \leq v(\|\phi-\psi\|)$, where $u, v \in \mathscr{P}=$ $\left\{u: R^{+} \rightarrow R^{+} \mid u\right.$ is continuous increasing function and $u(s) \rightarrow 0$, as $s \rightarrow 0\}$;

(2) $|V(t, \bar{\phi}, \bar{\psi})-V(t, \widehat{\phi}, \widehat{\psi})| \leq L(\|\bar{\phi}-\widehat{\phi}\|+\|\bar{\psi}-\widehat{\psi}\|)$, where $L$ is a positive constant;

(3) $\left.D^{+} V(t, \phi, \psi)\right|_{(15)} \leq-\gamma V(t, \phi, \psi)$, where $\gamma$ is a positive constant. Further, assume that (14) has a solution $x(t, v, \phi)$ such that $|x(t, v, \phi)| \leq B_{1}$ for $t \geq v \geq 0, B>$ $B_{1}>0$. Then system (14) has a unique almost periodic solution which is uniformly asymptotically stable.

Lemma 4 (see [37]). For any $x, y, u, v \in R$, one has the following inequality:

$$
|| x-y|-| u-v|| \leq|x-u|+|y-v| .
$$

Now, we will study the nonimpulsive system of system (3):

$$
\begin{aligned}
& u^{\prime}(t)=u(t)\left[r_{1}(t)-A(t) u\left(t-\tau_{1}\right)-B_{1}(t) v(t)\right. \\
& \left.-B_{2}(t) w(t)\right] \\
& v^{\prime}(t)=C(t) u\left(t-\tau_{2}\right) v\left(t-\tau_{2}\right)-v(t)\left[r_{2}(t)\right. \\
& \left.+D_{11}(t) v(t)+D_{12}(t) w(t)+E_{1}(t) w\left(t-\tau_{3}\right)\right] \\
& w^{\prime}(t)=E_{2}(t) v(t) w\left(t-\tau_{3}\right)-D_{21}(t) v(t) w(t) \\
& -D_{22}(t) w^{2}(t),
\end{aligned}
$$

with the initial condition

$$
\begin{aligned}
u(s) & =\phi_{1}(s), \\
v(s) & =\phi_{2}(s), \\
w(s) & =\phi_{3}(s), \\
0 & <u\left(s ; 0, \phi_{1}\right)=\phi_{1}(s)<+\infty, \\
0 & <v\left(s ; 0, \phi_{1}\right)=\phi_{2}(s)<+\infty, \\
0 & <w\left(s ; 0, \phi_{1}\right)=\phi_{3}(s)<+\infty,
\end{aligned}
$$

where $\phi_{i} \in P C(0), s \in[-\tau, 0], i=1,2,3$. Further expressions of functions $A(t), B_{j}(t), C(t), D_{j l}(t), E_{j}(t),(j, l=1,2)$ are given as follows:

$$
\begin{aligned}
A(t) & =H_{1}(t) a(t), \\
B_{1}(t) & =H_{2}(t) b_{1}(t), \\
B_{2}(t) & =H_{3}(t) b_{2}(t), \\
C(t) & =H_{1}(t) c(t), \\
E_{1}(t) & =H_{3}(t) e(t), \\
E_{2}(t) & =H_{2}(t) e(t), \\
D_{11}(t) & =H_{2}(t) d_{1}(t), \\
D_{12}(t) & =H_{3}(t) d_{1}(t),
\end{aligned}
$$




$$
\begin{aligned}
& D_{21}(t)=H_{2}(t) d_{2}(t), \\
& D_{22}(t)=H_{3}(t) d_{2}(t) .
\end{aligned}
$$

Then we have the following lemma.

Lemma 5. Assume that $(u(t), v(t), w(t))^{T}$ is any solution of system (17) with initial conditions (18); then $u(t)>0, v(t)>0$, $w(t)>0$ for all $t \in R^{+}$.

Proof. Let

$$
\begin{aligned}
M(t)= & r_{1}(t)-A(t) u\left(t-\tau_{1}\right)-B_{1}(t) v(t) \\
& -B_{2}(t) w(t), \\
N(t)= & r_{2}(t)+D_{11}(t) v(t)+D_{12}(t) w(t) \\
& +E_{1}(t) w\left(t-\tau_{3}\right), \\
P(t)= & D_{21} v(t)+D_{22} w(t) .
\end{aligned}
$$

Then it follows from the first equation of system (17) that

$$
\begin{aligned}
u(t) & =u(0) \exp \left\{\int_{0}^{t} M(s) d s\right\} \\
& =\phi_{1}(0) \exp \left\{\int_{0}^{t} M(s) d s\right\}>0 .
\end{aligned}
$$

From the second equation of system (17), we have

$$
v^{\prime}(t) \geq-v(t) N(t) \Longrightarrow \frac{v^{\prime}(t)}{v(t)} \geq-N(t)
$$

Integrating the above inequality on the interval $[0, t]$ yields

$$
\begin{aligned}
\ln v(t)-\ln v(0) & \geq-\int_{0}^{t} N(s) d s \Longrightarrow v(t) \\
& \geq \phi_{2}(0) \exp \left\{-\int_{0}^{t} N(s) d s\right\}>0 .
\end{aligned}
$$

Similarly, from the last equation of system (17), we have

$$
w^{\prime}(t) \geq-w(t) P(t) \Longrightarrow \frac{w^{\prime}(t)}{w(t)} \geq-P(t)
$$

Integrating the above inequality on the interval $[0, t]$ yields

$$
\begin{aligned}
\ln w(t)-\ln w(0) & \geq-\int_{0}^{t} P(s) d s \Longrightarrow w(t) \\
& \geq \phi_{3}(0) \exp \left\{-\int_{0}^{t} P(s) d s\right\}>0 .
\end{aligned}
$$

This completes the proof of this lemma.
Lemma 6. For system (3) and system (17), one has following results:

(1) If $(u(t), v(t), w(t))^{T}$ is a solution of system (17), then

$$
\begin{gathered}
(X(t), S(t), I(t))^{T}=\left(\prod_{0<t_{k}<t}\left(1+h_{1 k}\right) u(t),\right. \\
\left.\prod_{0<t_{k}<t}\left(1+h_{2 k}\right) v(t), \prod_{0<t_{k}<t}\left(1+h_{3 k}\right) w(t)\right)^{T}
\end{gathered}
$$

is a solution of impulsive system (3);

(2) If $(X(t), S(t), I(t))^{T}$ is a solution of system (3), then

$$
(u(t), v(t), w(t))^{T}=\left(\prod_{0<t_{k}<t}\left(1+h_{1 k}\right)^{-1} X(t),\right.
$$

$$
\left.\prod_{0<t_{k}<t}\left(1+h_{2 k}\right)^{-1} S(t), \prod_{0<t_{k}<t}\left(1+h_{3 k}\right)^{-1} I(t)\right)^{T}
$$

is a solution of the nonimpulsive system (17).

Proof. (1) If $(u(t), v(t), w(t))^{T}$ is a solution of system (17), then, for any $t \neq t_{k}, k \in Z$,

$$
\begin{aligned}
& u(t)=\prod_{0<t_{k}<t}\left(1+h_{1 k}\right)^{-1} X(t), \\
& v(t)=\prod_{0<t_{k}<t}\left(1+h_{2 k}\right)^{-1} S(t), \\
& w(t)=\prod_{0<t_{k}<t}\left(1+h_{3 k}\right)^{-1} I(t)
\end{aligned}
$$

is a solution of system (17), which yields

$$
\begin{aligned}
& \prod_{0<t_{k}<t}\left(1+h_{1 k}\right)^{-1} X^{\prime}(t)=\prod_{0<t_{k}<t}\left(1+h_{1 k}\right)^{-1} X(t) \\
& \quad \cdot\left\{r_{1}(t)\right. \\
& -A(t) \prod_{0<t_{k}<t}\left(1+h_{1 k}\right)^{-1} \times X\left(t-\tau_{1}\right) \\
& -B_{1}(t) \prod_{0<t_{k}<t}\left(1+h_{2 k}\right)^{-1} S(t) \\
& \left.-B_{2}(t) \prod_{0<t_{k}<t}\left(1+h_{3 k}\right)^{-1} I(t)\right\}
\end{aligned}
$$




$$
\begin{aligned}
& \prod_{0<t_{k}<t}\left(1+h_{2 k}\right)^{-1} S^{\prime}(t)=C(t) \\
& \cdot \prod_{0<t_{k}<t}\left(1+h_{1 k}\right)^{-1} X\left(t-\tau_{2}\right) \\
& \cdot \prod_{0<t_{k}<t}\left(1+h_{1 k}\right)^{-1} S\left(t-\tau_{2}\right) \\
& -\prod_{0<t_{k}<t}\left(1+h_{2 k}\right)^{-1} S(t)\left\{r_{2}(t)\right. \\
& +D_{11} \prod_{0<t_{k}<t}\left(1+h_{2 k}\right)^{-1} S(t) \\
& +D_{12} \prod_{0<t_{k}<t}\left(1+h_{3 k}\right)^{-1} I(t) \\
& \left.+E_{1}(t) \prod_{0<t_{k}<t}\left(1+h_{3 k}\right)^{-1} I\left(t-\tau_{3}\right)\right\}, \\
& \prod_{0<t_{k}<t}\left(1+h_{3 k}\right)^{-1} I^{\prime}(t)=E_{2}(t) \prod_{0<t_{k}<t}\left(1+h_{2 k}\right)^{-1} S(t) \\
& \cdot \prod_{0<t_{k}<t}\left(1+h_{3 k}\right)^{-1} I\left(t-\tau_{3}\right) \\
& -\prod_{0<t_{k}<t}\left(1+h_{3 k}\right)^{-1} I(t) \\
& \cdot\left[D_{21}(t) \prod_{0<t_{k}<t}\left(1+h_{2 k}\right)^{-1} S(t)\right. \\
& \left.+D_{22}(t) \prod_{0<t_{k}<t}\left(1+h_{3 k}\right)^{-1} I(t)\right] \text {. }
\end{aligned}
$$

By previous definitions of the function $A(t), B_{j}(t), C(t)$, $E_{j}(t)$ and $D_{j l}(t), j, l=1,2$, it follows from the simplification of (29) that

$$
\begin{aligned}
& X^{\prime}(t)=X(t)\left[r_{1}(t)-a(t) X\left(t-\tau_{1}\right)-b_{1}(t) S(t)\right. \\
& \left.\quad-b_{2}(t) I(t)\right] \\
& S^{\prime}(t)=c(t) X\left(t-\tau_{2}\right) S\left(t-\tau_{2}\right)+S(t)\left[-r_{2}(t)\right. \\
& \left.\quad-d_{1}(t)(S(t)+I(t))-e(t) I\left(t-\tau_{3}\right)\right], \\
& I^{\prime}(t)=e(t) S(t) I\left(t-\tau_{3}\right)-d_{2}(t)(S(t)+I(t)) I(t) .
\end{aligned}
$$

On the other hand, when $t=t_{k}, k \in N$, we have

$$
\begin{aligned}
X\left(t_{k}^{+}\right) & =\lim _{t \rightarrow t_{k}^{+}} \prod_{0<t_{k}<t}\left(1+h_{1 k}\right) u(t) \\
& =\prod_{0<t_{j} \leq t_{k}}\left(1+h_{1 j}\right) u\left(t_{j}\right) \\
& =\left(1+h_{1 k}\right) \prod_{0<t_{j}<t_{k}}\left(1+h_{1 j}\right) u\left(t_{j}\right) \\
& =\left(1+h_{1 k}\right) X\left(t_{k}\right) .
\end{aligned}
$$

Similarly, we can verify that

$$
\begin{aligned}
& S\left(t_{k}^{+}\right)=\left(1+h_{2 k}\right) S\left(t_{k}\right), \\
& I\left(t_{k}^{+}\right)=\left(1+h_{3 k}\right) I\left(t_{k}\right) .
\end{aligned}
$$

Thus, from (30)-(32) we know that $(X(t), S(t), I(t))^{T}$ is the solution of impulsive system (3).

(2) Since $u(t), v(t)$, and $w(t)$ are continuous on each interval $\left(t_{k}, t_{k+1}\right]$, then we only need to check the continuity of them at the impulsive point $t=t_{k}, k \in N$. In fact,

$$
\begin{aligned}
u\left(t_{k}^{+}\right) & =\prod_{0<t_{j} \leq t_{k}}\left(1+h_{1 j}\right)^{-1} X\left(t_{k}^{+}\right) \\
& =\prod_{0<t_{j}<t_{k}}\left(1+h_{1 j}\right)^{-1} X\left(t_{k}\right)=u\left(t_{k}\right), \\
u\left(t_{k}^{-}\right) & =\prod_{0<t_{j} \leq t_{k}}\left(1+h_{1 j}\right)^{-1} X\left(t_{k}^{-}\right) \\
& =\prod_{0<t_{j}<t_{k}}\left(1+h_{1 j}\right)^{-1} X\left(t_{k}\right)=u\left(t_{k}\right) .
\end{aligned}
$$

That is,

$$
u\left(t_{k}^{+}\right)=u\left(t_{k}^{-}\right)=u\left(t_{k}\right) .
$$

Similarly, we can check that

$$
\begin{gathered}
v\left(t_{k}^{+}\right)=v\left(t_{k}^{-}\right)=v\left(t_{k}\right), \\
w\left(t_{k}^{+}\right)=w\left(t_{k}^{-}\right)=w\left(t_{k}\right) .
\end{gathered}
$$

Therefore, $u(t), v(t)$, and $w(t)$ are continuous on $[0,+\infty)$.

On the other hand, similar to the above proof process of step (1), we can verify that $(u(t), v(t), w(t))^{T}$ satisfies system (17).

Then, $(u(t), v(t), w(t))^{T}$ is a solution of nonimpulsive system (17).

This completes the proof of Lemma 6.

\section{Main Results}

Theorem 7. Assume that (C1)-(C2) hold, and suppose further that

(C3) $C^{u} r_{1}^{u} e^{r_{1}^{u} \tau_{1}}>A^{l} r_{2}^{l}$;

(C4) $r_{1}^{l}>B_{1}^{u} v^{*}+B_{2}^{u} w^{*}$;

(C5) $C^{l} u_{*}>r_{2}^{u}+\left(D_{12}^{u}+E_{1}^{u}\right) w^{*}$;

(C6) $E_{2}^{l} v_{*}>D_{21}^{u} v^{*}$;

then any positive solution $(u(t), v(t), w(t))^{T}$ of system (17) satisfies

$$
\begin{aligned}
& u_{*} \leq \liminf _{t \rightarrow+\infty} u(t) \leq \limsup _{t \rightarrow+\infty} u(t) \leq u^{*}, \\
& v_{*} \leq \liminf _{t \rightarrow+\infty} v(t) \leq \limsup _{t \rightarrow+\infty} v(t) \leq v^{*}, \\
& w_{*} \leq \liminf _{t \rightarrow+\infty} w(t) \leq \limsup _{t \rightarrow+\infty} w(t) \leq w^{*},
\end{aligned}
$$


where

$$
\begin{aligned}
& u^{*}=\frac{r_{1}^{u}}{A^{l}} e^{r_{1}^{u} \tau_{1}} \\
& v^{*}=\frac{C^{u} r_{1}^{u} e^{r_{1}^{u} \tau_{1}}-A^{l} r_{2}^{l}}{A^{l} D_{11}^{l}}, \\
& w^{*}=\frac{E_{2}^{u}\left(C^{u} r_{1}^{u} e^{r_{1}^{u} \tau_{1}}-A^{l} r_{2}^{l}\right)}{A^{l} D_{11}^{l} D_{22}^{l}} \\
& u^{*}=\frac{r_{1}^{u}}{A^{l}} e^{r_{1}^{u} \tau_{1}}, \\
& v^{*}=\frac{C^{u} r_{1}^{u} e^{r_{1}^{u} \tau_{1}}-A^{l} r_{2}^{l}}{A^{l} D_{11}^{l}}, \\
& w^{*}=\frac{E_{2}^{u}\left(C^{u} r_{1}^{u} e^{r_{1}^{u} \tau_{1}}-A^{l} r_{2}^{l}\right)}{A^{l} D_{11}^{l} D_{22}^{l}}, \\
& u_{*}=\frac{r_{1}^{l}-\left(B_{1}^{u} v^{*}+B_{2}^{u} w^{*}\right)}{A^{u}} \\
& \cdot \exp \left\{\left(r_{1}^{l}-\left(B_{1}^{u} v^{*}+B_{2}^{u} w^{*}+A^{u} u^{*}\right)\right) \tau_{1}\right\} \text {, } \\
& v_{*}=\frac{\left(C^{l} u_{*}-r_{2}^{u}\right)-\left(D_{12}^{u}+E_{1}^{u}\right) w^{*}}{D_{11}^{u}} \text {, } \\
& w_{*}=\frac{E_{2}^{l} v_{*}-D_{21}^{u} v^{*}}{D_{22}^{u}} .
\end{aligned}
$$

Proof. From the first equation of system (17) we have

$$
u^{\prime}(t) \leq u(t)\left[r_{1}^{u}-A^{l} u\left(t-\tau_{1}\right)\right] .
$$

It follows from the first conclusion of Lemma 1 that

$$
\limsup _{t \rightarrow+\infty} u(t) \leq \frac{r_{1}^{u}}{A^{l}} e^{r_{1}^{u} \tau_{1}}=u^{*} .
$$

Thus, there exists a $T_{1}>0$, such that $u(t) \leq u^{*}$ when $t>T_{1}$.

At the moment, from the second equation of system (17), when $t>T_{1}+\tau_{2}$, we have

$$
\begin{aligned}
v^{\prime}(t) & \leq c^{u} u^{*} v\left(t-\tau_{2}\right)-v(t)\left(r_{2}^{l}+D_{11}^{l} v(t)\right) \\
& =c^{u} u^{*} v\left(t-\tau_{2}\right)-r_{2}^{l} v(t)-D_{11}^{l} v^{2}(t) .
\end{aligned}
$$

Since condition (C3) holds, then it follows from the second conclusion of Lemma 2 that

$$
\limsup _{t \rightarrow+\infty} v(t) \leq \frac{c^{u} u^{*}-r_{2}^{l}}{D_{11}^{l}}=\frac{C^{u} r_{1}^{u} e^{r_{1}^{u} \tau_{1}}-A^{l} r_{2}^{l}}{A^{l} D_{11}^{l}}=v^{*}
$$

Therefore, there exists a $T_{2}>T_{1}+\tau_{2}$, such that $v(t) \leq$ $v^{*}$ when $t>T_{2}$, and when $t>T_{2}$ from the last equation of system (17) we have

$$
w^{\prime}(t) \leq E_{2}^{u} v^{*} w\left(t-\tau_{3}\right)-D_{22}^{l} w^{2}(t) .
$$

Then it follows from the second conclusion of Lemma 2 again that

$$
\begin{aligned}
\limsup _{t \rightarrow+\infty} w(t) & \leq \frac{E_{2}^{u} v^{*}}{D_{22}^{l}}=\frac{E_{2}^{u}\left(C^{u} r_{1}^{u} e^{r_{1}^{u} \tau_{1}}-A^{l} r_{2}^{l}\right)}{A^{l} D_{11}^{l} D_{22}^{l}} \\
& =w^{*} .
\end{aligned}
$$

Therefore, there exists a $T_{3}>T_{2}$, such that $w(t) \leq w^{*}$ when $t>T_{3}$.

At the moment, considering the inequality estimations on the opposite direction from all the equations of system (17), we can obtain

$$
u^{\prime}(t) \geq u(t)\left[r_{1}^{l}-A^{u} u\left(t-\tau_{1}\right)-\left(B_{1}^{u} v^{*}+B_{2}^{u} w^{*}\right)\right] .
$$

Since condition (C4) holds, then by the second conclusion of Lemma 1 we have

$$
\begin{aligned}
& \liminf _{t \rightarrow+\infty} u(t) \geq \frac{r_{1}^{l}-\left(B_{1}^{u} v^{*}+B_{2}^{u} w^{*}\right)}{A^{u}} \\
& \quad \cdot \exp \left\{\left(r_{1}^{l}-\left(B_{1}^{u} v^{*}+B_{2}^{u} w^{*}+A^{u} u^{*}\right)\right) \tau_{1}\right\}=u_{*} .
\end{aligned}
$$

Then there exists a $T_{4}>T_{3}$, such that $u(t) \geq u_{*}$ when $t>T_{4}$. When $t>T_{4}+\tau_{2}$ it follows from the second equation of system (17) that

$$
\begin{aligned}
v(t) \geq & C^{l} u_{*} v\left(t-\tau_{2}\right) \\
& -v(t)\left[r_{2}^{u}+D_{11}^{u} v(t)+\left(D_{12}^{u}+E_{1}^{u}\right) w^{*}\right] .
\end{aligned}
$$

Since condition (C5) holds, and by the second conclusion of Lemma 2 again, we have

$$
\liminf _{t \rightarrow+\infty} v(t) \geq \frac{\left(C^{l} u-r_{2}^{u}\right)-\left(D_{12}^{u}+E_{1}^{u}\right) w^{*}}{D_{11}^{u}}=v_{*} .
$$

Then there exists a $T_{5}>T_{4}+\tau_{2}$, such that $v(t) \geq v_{*}$ when $t>T_{5}$. When $t>T_{5}$, it follows from the last equation of system (17) that

$$
w(t) \geq E_{2}^{l} v_{*} w\left(t-\tau_{3}\right)-D_{21}^{u} v^{*} w(t)-D_{22}^{u} w^{2}(t) .
$$

Since condition (C6) holds and it follows from Lemma 2 again, we have that

$$
\liminf _{t \rightarrow+\infty} w(t) \geq \frac{E_{2}^{l} v_{*}-D_{21}^{u} v^{*}}{D_{22}^{u}}=w_{*} .
$$

Thus, combining (39), (41), and (43) with (45), (47), and (49), we can see that the proof of this theorem is completed.

Theorem 8. Assume that (C1)-(C6) hold; then any positive solution $(X(t), S(t), I(t))^{T}$ of system (3) satisfies

$$
\begin{aligned}
& H_{1}^{l} u_{*} \leq \liminf _{t \rightarrow+\infty} X(t) \leq \limsup _{t \rightarrow+\infty} X(t) \leq H_{1}^{u} u^{*}, \\
& H_{2}^{l} v_{*} \leq \liminf _{t \rightarrow+\infty} S(t) \leq \limsup _{t \rightarrow+\infty} S(t) \leq H_{2}^{u} v^{*} ; \\
& H_{3}^{l} w_{*} \leq \liminf _{t \rightarrow+\infty} I(t) \leq \limsup _{t \rightarrow+\infty} I(t) \leq H_{3}^{u} w^{*} .
\end{aligned}
$$


Proof. Since $(X(t), S(t), I(t))^{T}$ is a solution of system (3), then by the second conclusion of Lemma 6

$$
(u(t), v(t), w(t))^{T}=\left(\frac{X(t)}{H_{1}(t)}, \frac{S(t)}{H_{2}(t)}, \frac{I(t)}{H_{3}(t)}\right)^{T}
$$

is a solution of system (17).

Then it follows from Theorem 7 that

$$
\begin{aligned}
u_{*} & \leq \liminf _{t \rightarrow+\infty} \frac{X(t)}{H_{1}(t)} \leq \liminf _{t \rightarrow+\infty} u(t) \leq \limsup _{t \rightarrow+\infty} u(t) \\
& =\limsup _{t \rightarrow+\infty} \frac{X(t)}{H_{1}(t)} \leq u^{*}, \\
v_{*} & \leq \liminf _{t \rightarrow+\infty} \frac{S(t)}{H_{2}(t)} \leq \liminf _{t \rightarrow+\infty} v(t) \leq \limsup _{t \rightarrow+\infty} v(t) \\
& =\limsup _{t \rightarrow+\infty} \frac{S(t)}{H_{2}(t)} \leq v^{*}, \\
w_{*} & \leq \liminf _{t \rightarrow+\infty} \frac{I(t)}{H_{3}(t)} \leq \liminf _{t \rightarrow+\infty} w(t) \leq \limsup _{t \rightarrow+\infty} w(t) \\
& =\limsup _{t \rightarrow+\infty} \frac{I(t)}{H_{3}(t)} \leq w^{*},
\end{aligned}
$$

which implies that

$$
\begin{aligned}
& H_{1}^{l} u_{*} \leq \liminf _{t \rightarrow+\infty} X(t) \leq \limsup _{t \rightarrow+\infty} X(t) \leq H_{1}^{u} u^{*}, \\
& H_{2}^{l} v_{*} \leq \liminf _{t \rightarrow+\infty} S(t) \leq \limsup _{t \rightarrow+\infty} S(t) \leq H_{2}^{u} v^{*} \\
& H_{3}^{l} w_{*} \leq \liminf _{t \rightarrow+\infty} I(t) \leq \limsup _{t \rightarrow+\infty} I(t) \leq H_{3}^{u} w^{*} .
\end{aligned}
$$

This completes the proof of this theorem.

Remark 9. Suppose that (C1)-(C6) hold; then system (3) is permanent.

In the following, we will discuss the uniformly asymptotic stability of a unique almost periodic solution of system (3) by Lemma 4 . And for the sake of convenience, we give some notations before the theorem as follows.

Theorem 10. Assume that (C1)-(C6) hold, and further assume that

(C7) there exists a set of positive parameters $\lambda_{i}>0(i=$ $1,2,3)$, such that the following linear matrix inequality holds:

$$
\left(\begin{array}{lll}
Q_{11} & Q_{12} & Q_{13} \\
Q_{21} & Q_{22} & Q_{23} \\
Q_{31} & Q_{32} & Q_{33}
\end{array}\right)\left(\begin{array}{c}
\lambda_{1} \\
\lambda_{2} \\
\lambda_{3}
\end{array}\right)>0
$$

Then system (3) admits a unique almost periodic solution which is uniformly asymptotically stable, where

$$
\begin{aligned}
& Q_{11}=A_{1}^{l} u_{*}-\tau_{1}\left(A^{u} u^{*}\right)^{2}, \\
& Q_{12}=-\tau_{2}\left(\frac{C^{u} u^{*} v^{*}}{v_{*}}\right)^{2}-\frac{C^{u} u^{*} v^{*}}{v_{*}}, \\
& Q_{13}=0 \text {, } \\
& Q_{21}=-B_{1}^{u} v^{*}-\tau_{1} A^{u} B_{1}^{u} u^{*} v^{*}, \\
& Q_{22}=D_{11}^{l} v_{*}-2 \tau_{2}\left(\frac{C^{u} u^{*} v^{*}}{v_{*}}\right)^{2}-\tau_{2} \frac{C^{u} D_{11}^{u} u^{*}\left(v^{*}\right)^{2}}{v_{*}} \text {, } \\
& Q_{23}=-\frac{E_{2}^{u} v^{*} w^{*}}{w_{*}}-D_{21}^{u} v^{*}-\tau_{3} \frac{D_{21}^{u} E_{2}^{u}\left(v^{*}\right)^{2} w^{*}}{w_{*}} \\
& -\tau_{3}\left(\frac{E_{2}^{u} v^{*} w^{*}}{w_{*}}\right)^{2} \\
& Q_{31}=-B_{2}^{u} w^{*}-\tau_{1} A^{u} B_{2}^{u} u^{*} w^{*} \text {, } \\
& Q_{32}=-D_{12}^{u} w^{*}-E_{1}^{u} w^{*}-\tau_{2} \frac{C^{u} D_{12}^{u} u^{*} v^{*} w^{*}}{v_{*}} \\
& -\tau_{2} \frac{C^{u} E_{1}^{u} u^{*} v^{*} w^{*}}{v_{*}} \\
& Q_{33}=D_{22}^{l} w_{*}-2 \tau_{3}\left(\frac{E_{2}^{u} v^{*} w^{*}}{w_{*}}\right)^{2} \\
& -\tau_{3} \frac{D_{22}^{u} E_{2}^{u}\left(w^{*}\right)^{2} v^{*}}{w_{*}} .
\end{aligned}
$$

Proof. At first, we will prove that system (17) has a unique uniformly asymptotically stable almost periodic solution. In order to achieve this aim, we take a transformation:

$$
\begin{gathered}
u(t)=e^{x_{1}(t),} \\
v(t)=e^{s_{1}(t)}, \\
w(t)=e^{i_{1}(t)},
\end{gathered}
$$

$$
t \in R^{+}
$$

Then system (17) is transformed into the following system:

$$
\begin{aligned}
& x_{1}^{\prime}(t)=r_{1}(t)-A(t) e^{x_{1}\left(t-\tau_{1}\right)}-B_{1}(t) e^{s_{1}(t)}-B_{2}(t) \\
& \cdot e^{i_{1}(t)}, \\
& s_{1}^{\prime}(t)=C(t) e^{x_{1}\left(t-\tau_{2}\right)} e^{s_{1}\left(t-\tau_{2}\right)-s_{1}(t)}-\left[r_{2}(t)\right. \\
& \left.\quad+D_{11}(t) e^{s_{1}(t)}+D_{12}(t) e^{i_{1}(t)}+E_{1}(t) e^{i_{1}\left(t-\tau_{3}\right)}\right], \\
& i_{1}^{\prime}(t)=E_{2}(t) e^{s_{1}(t)} e^{i_{1}\left(t-\tau_{3}\right)-i_{1}(t)}-\left[D_{21}(t) e^{s_{1}(t)}\right. \\
& \left.\quad+D_{22}(t) e^{i_{1}(t)}\right] .
\end{aligned}
$$


Suppose that $U_{1}(t)=\left(x_{1}(t), s_{1}(t), i_{1}(t)\right)^{T}$ and $U_{2}(t)=$ $\left(x_{2}(t), s_{2}(t), i_{2}(t)\right)^{T}$ are any two solutions of system (59); then the product system of (59) reads

$$
\begin{aligned}
& x_{1}^{\prime}(t)=r_{1}(t)-A(t) e^{x_{1}\left(t-\tau_{1}\right)}-B_{1}(t) e^{s_{1}(t)}-B_{2}(t) \\
& \cdot e^{i_{1}(t)}, \\
& s_{1}^{\prime}(t)=C(t) e^{x_{1}\left(t-\tau_{2}\right)} e^{s_{1}\left(t-\tau_{2}\right)-s_{1}(t)}-\left[r_{2}(t)\right. \\
& \left.\quad+D_{11}(t) e^{s_{1}(t)}+D_{12}(t) e^{i_{1}(t)}+E_{1}(t) e^{i_{1}\left(t-\tau_{3}\right)}\right] \\
& i_{1}^{\prime}(t)=E_{2}(t) e^{s_{1}(t)} e^{i_{1}\left(t-\tau_{3}\right)-i_{1}(t)}-\left[D_{21}(t) e^{s_{1}(t)}\right. \\
& \left.+D_{22}(t) e^{i_{1}(t)}\right] . \\
& x_{2}^{\prime}(t)=r_{1}(t)-A(t) e^{x_{2}\left(t-\tau_{1}\right)}-B_{1}(t) e^{s_{2}(t)}-B_{2}(t) \\
& \cdot e^{i_{2}(t)}, \\
& s_{2}^{\prime}(t)=C(t) e^{x_{2}\left(t-\tau_{2}\right)} e^{s_{2}\left(t-\tau_{2}\right)-s_{2}(t)}-\left[r_{2}(t)\right. \\
& \left.\quad+D_{11}(t) e^{s_{2}(t)}+D_{12}(t) e^{i_{2}(t)}+E_{1}(t) e^{i_{2}\left(t-\tau_{3}\right)}\right] \\
& i_{2}^{\prime}(t)=E_{2}(t) e^{s_{2}(t)} e^{i_{2}\left(t-\tau_{3}\right)-i_{2}(t)}-\left[D_{21}(t) e^{s_{2}(t)}\right. \\
& \left.+D_{22}(t) e^{i_{2}(t)}\right] .
\end{aligned}
$$

Denote $S^{*}=\left\{\phi=\left(x_{1 t}, s_{1 t}, i_{1 t}\right)^{T} \in C\left([-\tau, 0], R^{3}\right) \mid \ln u_{*} \leq\right.$ $\left.x_{1 t} \leq \ln u^{*}, \ln v_{*} \leq s_{1 t} \leq \ln v^{*}, \ln w_{*} \leq i_{1 t} \leq \ln w^{*}, t \in R^{+}\right\}$.

For any $\Phi=\left(\phi_{1}, \phi_{2}, \phi_{3}\right)^{T}=\left(x_{1 t}, s_{1 t}, i_{1 t}\right)^{T} \in S^{*}$, we can choose $\Psi=\left(\psi_{1}, \psi_{2}, \psi_{3}\right)^{T}=\left(x_{2 t}, s_{2 t}, i_{2 t}\right)^{T} \in S^{*}$ such that

$$
\begin{aligned}
|\Phi(0)-\Psi(0)|_{0}= & \left|\phi_{1}(0)-\psi_{1}(0)\right|+\left|\phi_{2}(0)-\psi_{2}(0)\right| \\
& +\left|\phi_{3}(0)-\psi_{3}(0)\right|>0 .
\end{aligned}
$$

Consider a Lyapunov functional $V(t)=V(t, \Phi, \Psi)=$ $V\left(t,\left(x_{1 t}, s_{1 t}, i_{1 t}\right)^{T},\left(x_{2 t}, s_{2 t}, i_{2 t}\right)^{T}\right)=V_{1}(t)+V_{2}(t)+V_{3}(t)+$ $V_{4}(t)+V_{5}(t)$ defined on $R^{+} \times S^{*} \times S^{*}$, where

$$
\begin{aligned}
& V_{1}(t)=\lambda_{1}\left|x_{1}(t)-x_{2}(t)\right|+\lambda_{2}\left|s_{1}(t)-s_{2}(t)\right| \\
& \quad+\lambda_{3}\left|i_{1}(t)-i_{2}(t)\right| ; \\
& V_{2}(t)=\lambda_{1}\left(A^{u} u^{*}\right)^{2} \int_{-2 \tau_{1}}^{-\tau_{1}} \int_{t+s}^{t}\left|x_{1}(r)-x_{2}(r)\right| d r d s \\
& \quad+\lambda_{2}\left(\frac{C^{u} u^{*} v^{*}}{v_{*}}\right)^{2} \int_{-2 \tau_{2}}^{-\tau_{2}} \int_{t+s}^{t}\left|x_{1}(r)-x_{2}(r)\right| d r d s
\end{aligned}
$$

$$
\begin{aligned}
& V_{3}(t)=\lambda_{1} A^{u} B_{1}^{u} u^{*} v^{*} \int_{-\tau_{1}}^{0} \int_{t+s}^{t}\left|s_{1}(r)-s_{2}(r)\right| d r d s \\
& +\lambda_{2}\left(\frac{C^{u} u^{*} v^{*}}{v_{*}}\right)^{2} \int_{-2 \tau_{2}}^{0} \int_{t+s}^{t}\left|s_{1}(r)-s_{2}(r)\right| d r d s \\
& +\lambda_{2} \frac{C^{u} D_{11}^{u} u^{*}\left(v^{*}\right)^{2}}{v_{*}} \int_{-\tau_{2}}^{0} \int_{t+s}^{t}\left|s_{1}(r)-s_{2}(r)\right| d r d s \\
& +\lambda_{3}\left(\frac{E_{2}^{u} v^{*} w^{*}}{w_{*}}\right)^{2} \int_{-\tau_{3}}^{0} \int_{t+s}^{t}\left|s_{1}(r)-s_{2}(r)\right| d r d s \\
& +\lambda_{3} \frac{D_{21}^{u} E_{2}^{u}\left(v^{*}\right)^{2} w^{*}}{w_{*}} \\
& +\int_{-\tau_{3}}^{0} \int_{t+s}^{t}\left|s_{1}(r)-s_{2}(r)\right| d r d s ; \\
& V_{4}(t)=\lambda_{1} A^{u} B_{2}^{u} u^{*} w^{*} \int_{-\tau_{1}}^{0} \int_{t+s}^{t}\left|i_{1}(r)-i_{2}(r)\right| d r d s \\
& +\lambda_{2} \frac{C^{u} D_{12}^{u} u^{*} v^{*} w^{*}}{v_{*}} \int_{-\tau_{2}}^{0} \int_{t+s}^{t}\left|i_{1}(r)-i_{2}(r)\right| d r d s \\
& +\lambda_{2} E_{1}^{u} w^{*} \int_{t-\tau_{3}}^{t}\left|i_{1}(s)-i_{2}(s)\right| d s . \\
& +\lambda_{2} \frac{C^{u} E_{1}^{u} u^{*} v^{*} w^{*}}{v_{*}} \int_{-\tau_{2}-\tau_{3}}^{-\tau_{3}} \int_{t+s}^{t}\left|i_{1}(r)-i_{2}(r)\right| d r d s \\
& +\lambda_{3}\left(\frac{E_{2}^{u} v^{*} w^{*}}{w_{*}}\right)^{2} \int_{-2 \tau_{3}}^{0} \int_{t+s}^{t}\left|i_{1}(r)-i_{2}(r)\right| d r d s \\
& w_{*}
\end{aligned}
$$

According to the definitions of $S^{*}$ and $V(t)=V(t, \Phi, \Psi)$, there is some positive constant $M$ large enough such that

$$
V(t)=V(t, \Phi, \Psi) \leq M
$$

By the structure of $V(t)$, it is easy to see

$$
\begin{aligned}
& V(t) \geq V_{1}(t) \geq \min \left\{\lambda_{1}, \lambda_{2}, \lambda_{3}\right\}\left(\left|x_{1}(t)-x_{2}(t)\right|\right. \\
& \left.+\left|s_{1}(t)-s_{2}(t)\right|+\left|i_{1}(t)-i_{2}(t)\right|\right) .
\end{aligned}
$$

Let $\underline{\lambda}=\min \left\{\lambda_{1}, \lambda_{2}, \lambda_{3}\right\}>0$, and then

$$
V(t) \geq V_{1}(t) \geq \underline{\lambda}|\Phi(0)-\Psi(0)|_{0}>0 .
$$


Moreover, by the integrative inequality and the absolutevalue inequality properties we have

$$
\begin{aligned}
& V(t) \leq \frac{1}{2}\left\{2\left(\lambda_{1}+\lambda_{2}+\lambda_{3}\right)+\tau^{2}\left[\lambda_{1}\left(A^{u} u^{*}\right)^{2}\right.\right. \\
& +\lambda_{1} A^{u} B_{1}^{u} u^{*} v^{*}+\lambda_{1} A^{u} B_{2}^{u} u^{*} w^{*}+\lambda_{2}\left(\frac{C^{u} u^{*} v^{*}}{v_{*}}\right)^{2} \\
& +\lambda_{2}\left(\frac{C^{u} u^{*} v^{*}}{v_{*}}\right)^{2}+\lambda_{2} \frac{C^{u} D_{11}^{u} u^{*}\left(v^{*}\right)^{2}}{v_{*}} \\
& +\lambda_{2} \frac{C^{u} D_{12}^{u} u^{*} v^{*} w^{*}}{v_{*}}+\lambda_{2} \frac{C^{u} E_{1}^{u} u^{*} v^{*} w^{*}}{v_{*}} \\
& +\lambda_{3}\left(\frac{E_{2}^{u} v^{*} w^{*}}{w_{*}}\right)^{2}+\lambda_{3} \frac{D_{21}^{u} E_{2}^{u}\left(v^{*}\right)^{2} w^{*}}{w_{*}} \\
& \left.+\lambda_{3}\left(\frac{E_{2}^{u} v^{*} w^{*}}{w_{*}}\right)^{2}+\lambda_{3} \frac{D_{22}^{u} E_{2}^{u}\left(w^{*}\right)^{2} v^{*}}{w_{*}}\right] \\
& \left.+\tau\left[\frac{\lambda_{2} C^{u} u^{*} v^{*}}{v_{*}}+\lambda_{2} E_{1}^{u} w^{*}\right]\right\} \\
& \left.+i_{1 t}(s)-i_{2 t}(s) \mid\right]=\bar{\lambda}\|\Phi-\Psi\| \\
& +\sup _{s \in[-\tau, 0]}\left[\left|x_{1 t}(s)-x_{2 t}(s)\right|+\left|s_{1 t}(s)-s_{2 t}(s)\right|\right.
\end{aligned}
$$

where

$$
\begin{aligned}
\bar{\lambda}: & =\frac{1}{2}\left\{2\left(\lambda_{1}+\lambda_{2}+\lambda_{3}\right)+\tau^{2}\left[\lambda_{1}\left(A^{u} u^{*}\right)^{2}\right.\right. \\
& +\lambda_{1} A^{u} B_{1}^{u} u^{*} v^{*}+\lambda_{1} A^{u} B_{2}^{u} u^{*} w^{*}+\lambda_{2}\left(\frac{C^{u} u^{*} v^{*}}{v_{*}}\right)^{2} \\
& +\lambda_{2}\left(\frac{C^{u} u^{*} v^{*}}{v_{*}}\right)^{2}+\lambda_{2} \frac{C^{u} D_{11}^{u} u^{*}\left(v^{*}\right)^{2}}{v_{*}} \\
& +\lambda_{2} \frac{C^{u} D_{12}^{u} u^{*} v^{*} w^{*}}{v_{*}}+\lambda_{2} \frac{C^{u} E_{1}^{u} u^{*} v^{*} w^{*}}{v_{*}} \\
& +\lambda_{3}\left(\frac{E_{2}^{u} v^{*} w^{*}}{w_{*}}\right)^{2}+\lambda_{3} \frac{D_{21}^{u} E_{2}^{u}\left(v^{*}\right)^{2} w^{*}}{w_{*}} \\
& \left.+\lambda_{3}\left(\frac{E_{2}^{u} v^{*} w^{*}}{w_{*}}\right)^{2}+\lambda_{3} \frac{D_{22}^{u} E_{2}^{u}\left(w^{*}\right)^{2} v^{*}}{w_{*}}\right] \\
& \left.+\tau\left[\frac{\lambda_{2} C^{u} u^{*} v^{*}}{v_{*}}+\lambda_{2} E_{1}^{u} w^{*}\right]\right\}
\end{aligned}
$$

Let $u, v \in C\left(R^{+}, R^{+}\right)$, choose $u=\underline{\lambda} s, v=\bar{\lambda} s$, and then the first condition of Lemma 3 is satisfied.

For any $\bar{\Phi}=\left(x_{1 t}, s_{1 t}, i_{1 t}\right)^{T}, \bar{\Psi}=\left(x_{2 t}, s_{2 t}, i_{2 t}\right)^{T}, \widehat{\Phi}=$ $\left(x_{1 t}^{*}, s_{1 t}^{*}, i_{1 t}^{*}\right)^{T}, \widehat{\Psi}=\left(x_{2 t}^{*}, s_{2 t}^{*}, i_{2 t}^{*}\right)^{T} \in S^{*}$, it follows from the previous definitions of $V_{i}(t)(i=1,2,3,4,5)$, and by inequality (16) in Lemma 4 we have that,

$$
\begin{aligned}
& |V(t, \bar{\Phi}, \bar{\Psi})-V(t, \widehat{\Phi}, \widehat{\Psi})| \leq \lambda_{1}|| x_{1}(t)-x_{1}^{*}(t) \mid \\
& \quad+\left|x_{2}(t)-x_{2}^{*}(t) \|+\lambda_{2}\right|\left|s_{1}(t)-s_{1}^{*}(t)\right|+\mid s_{2}(t) \\
& \quad-s_{2}^{*}(t)\left\|+\lambda_{3}|| i_{1}(t)-i_{1}^{*}(t)|+| i_{2}(t)-i_{2}^{*}(t)\right\| \\
& \quad+\lambda_{1}\left(A^{u} u^{*}\right)^{2} \int_{-2 \tau_{1}}^{-\tau_{1}} \int_{t+s}^{t}|| x_{1}(r)-x_{1}^{*}(r) \mid \\
& +\mid x_{2}(r)-x_{2}^{*}(r) \| d r d s+\lambda_{2}\left(\frac{C^{u} u^{*} v^{*}}{v_{*}}\right)^{2} \\
& \quad \cdot \int_{-2 \tau_{2}}^{-\tau_{2}} \int_{t+s}^{t}|| s_{1}(t)-s_{2}(t)|+| s_{1}^{*}(t)-s_{2}^{*}(t) \| d r d s \\
& \quad+\lambda_{1} A^{u} B_{1}^{u} u^{*} v^{*} \int_{-\tau_{1}}^{0} \int_{t+s}^{t}|| s_{1}(t)-s_{1}^{*}(t) \mid \\
& \quad+\mid s_{2}(t)-s_{2}^{*}(t) \| d r d s+\lambda_{2}\left(\frac{C^{u} u^{*} v^{*}}{v_{*}}\right)^{2} \\
& \quad+\lambda_{-2 \tau_{2}}^{0} \int_{t+s}^{t}\left\|s_{1}(t)-s_{1}^{*}(t)|+| s_{2}(t)-s_{2}^{*}(t)\right\| d r d s \\
& \quad D_{11}^{u} u^{*}\left(v^{*}\right)^{2} \int_{-\tau_{2}}^{0} \int_{t+s}^{t} \| s_{1}(t)-s_{1}^{*}(t) \mid
\end{aligned}
$$

$+\left|s_{2}(t)-s_{2}^{*}(t)\right| \mid d r d s+\lambda_{3}\left(\frac{E_{2}^{u} v^{*} w^{*}}{w_{*}}\right)^{2}$

$\cdot \int_{-\tau_{3}}^{0} \int_{t+s}^{t}\left\|s_{1}(t)-s_{1}^{*}(t)|+| s_{2}(t)-s_{2}^{*}(t)\right\| d r d s$

$+\lambda_{3} \frac{D_{21}^{u} E_{2}^{u}\left(v^{*}\right)^{2} w^{*}}{w_{*}} \int_{-\tau_{3}}^{0} \int_{t+s}^{t}|| s_{1}(t)-s_{1}^{*}(t) \mid$

$+\left|s_{2}(t)-s_{2}^{*}(t)\right| \mid d r d s$

$+\lambda_{1} A^{u} B_{2}^{u} u^{*} w^{*} \int_{-\tau_{1}}^{0} \int_{t+s}^{t}|| i_{1}(t)-i_{1}^{*}(t) \mid$

$+\left|i_{2}(t)-i_{2}^{*}(t)\right| \mid d r d s+\lambda_{2} \frac{C^{u} D_{12}^{u} u^{*} v^{*} w^{*}}{v_{*}}$

$\cdot \int_{-\tau_{2}}^{0} \int_{t+s}^{t}\left\|i_{1}(t)-i_{1}^{*}(t)|+| i_{2}(t)-i_{2}^{*}(t)\right\| d r d s$

$+\lambda_{2} \frac{C^{u} E_{1}^{u} u^{*} v^{*} w^{*}}{v_{*}} \int_{-\tau_{2}-\tau_{3}}^{-\tau_{3}} \int_{t+s}^{t}|| i_{1}(t)-i_{1}^{*}(t) \mid$

$+\mid i_{2}(t)-i_{2}^{*}(t) \| d r d s+\lambda_{3}\left(\frac{E_{2}^{u} v^{*} w^{*}}{w_{*}}\right)^{2}$

$\cdot \int_{-2 \tau_{3}}^{0} \int_{t+s}^{t}\left\|i_{1}(t)-i_{1}^{*}(t)|+| i_{2}(t)-i_{2}^{*}(t)\right\| d r d s$ 


$$
\begin{aligned}
& +\lambda_{3} \frac{D_{22}^{u} E_{2}^{u}\left(w^{*}\right)^{2} v^{*}}{w_{*}} \int_{-\tau_{3}}^{0} \int_{t+s}^{t}|| i_{1}(t)-i_{1}^{*}(t) \mid \\
& +\left|i_{2}(t)-i_{2}^{*}(t)\right| \mid d r d s+\lambda_{2} \frac{C^{u} u^{*} v^{*}}{v_{*}} \\
& \cdot \int_{t-\tau_{2}}^{t}\left\|x_{1}(s)-x_{1}^{*}(s)|+| x_{2}(s)-x_{2}^{*}(s)\right\| d s \\
& +\lambda_{2} E_{1}^{u} w^{*} \int_{t-\tau_{3}}^{t}|| i_{1}(s)-i_{1}^{*}(s)|+| i_{2}(s) \\
& -i_{2}^{*}(s) \| d s .
\end{aligned}
$$

This yields

$$
\begin{aligned}
& |V(t, \bar{\Phi}, \bar{\Psi})-V(t, \widehat{\Phi}, \widehat{\Psi})| \\
& \quad \leq \bar{\lambda} \sum_{i=1}^{2} \sup _{s \in[-\tau, 0]}\left[\left|x_{i t}(s)-x_{i t}^{*}(s)\right|+\left|s_{i t}(s)-s_{i t}^{*}(s)\right|\right. \\
& \left.\quad+\left|i_{i t}(s)-i_{i t}^{*}(s)\right|\right]=\bar{\lambda}(\|\bar{\Phi}-\widehat{\Phi}\|+\|\bar{\Psi}-\widehat{\Psi}\|) .
\end{aligned}
$$

This means the second condition of Lemma 3 is satisfied. Finally, we will prove the last condition of Lemma 3.

In fact, calculating the right derivative $D^{+} V_{1}(t)$ of $V_{1}(t)$ along the solutions of system (60) leads to

$$
\begin{aligned}
& D^{+} V_{1}(t)=\left(x_{1}^{\prime}(t)-x_{2}^{\prime}(t)\right) \operatorname{sgn}\left(x_{1}(t)-x_{2}(t)\right) \\
& \quad+\left(s_{1}^{\prime}(t)-s_{2}^{\prime}(t)\right) \operatorname{sgn}\left(s_{1}(t)-s_{2}(t)\right) \\
& \quad+\left(i_{1}^{\prime}(t)-i_{2}^{\prime}(t)\right) \operatorname{sgn}\left(i_{1}(t)-i_{2}(t)\right) .
\end{aligned}
$$

It follows from the mean-value theorem and the product system (60) that

$$
\begin{aligned}
x_{1}^{\prime}(t) & -x_{2}^{\prime}(t) \\
= & -A(t)\left[e^{x_{1}\left(t-\tau_{1}\right)}-e^{x_{2}\left(t-\tau_{1}\right)}\right] \\
& -B_{1}(t)\left[e^{s_{1}(t)}-e^{s_{2}(t)}\right]-B_{2}(t)\left[e^{i_{1}(t)}-e^{i_{2}(t)}\right] \\
= & -A(t) e^{\theta_{11}(t)}\left[x_{1}\left(t-\tau_{1}\right)-x_{2}\left(t-\tau_{1}\right)\right] \\
& -B_{1}(t) e^{\theta_{12}(t)}\left[s_{1}(t)-s_{2}(t)\right] \\
& -B_{2}(t) e^{\theta_{13}(t)}\left[i_{1}(t)-i_{2}(t)\right],
\end{aligned}
$$

where $\theta_{11}(t)$ lie between $x_{1}\left(t-\tau_{1}\right)$ and $x_{2}\left(t-\tau_{1}\right), \theta_{12}(t)$ lie between $s_{1}(t)$ and $s_{2}(t)$, and $\theta_{13}(t)$ lie between $i_{1}(t)$ and $i_{2}(t)$.

Thus,

$$
\begin{aligned}
x_{1}^{\prime}(t) & -x_{2}^{\prime}(t) \\
= & -A(t) e^{\theta_{11}(t)}\left[x_{1}(t)-x_{2}(t)\right] \\
& +A(t) e^{\theta_{11}(t)} \int_{t-\tau_{1}}^{t}\left[x_{1}^{\prime}(s)-x_{2}^{\prime}(s)\right] d s \\
& -B_{1}(t) e^{\theta_{12}(t)}\left[s_{1}(t)-s_{2}(t)\right] \\
& -B_{2}(t) e^{\theta_{13}(t)}\left[i_{1}(t)-i_{2}(t)\right] .
\end{aligned}
$$

Integrating both sides of (71) on the interval $\left[t-\tau_{1}, t\right]$, we have

$$
\begin{aligned}
& \int_{t-\tau_{1}}^{t}\left[x_{1}^{\prime}(s)-x_{2}^{\prime}(s)\right] d s=-\int_{t-\tau_{1}}^{t} A(s) \\
& \cdot e^{\theta_{11}(s)}\left[x_{1}\left(s-\tau_{1}\right)-x_{2}\left(s-\tau_{1}\right)\right] d s \\
& -\int_{t-\tau_{1}}^{t} B_{1}(s) e^{\theta_{12}(s)}\left[s_{1}(s)-s_{2}(s)\right] d s \\
& -\int_{t-\tau_{1}}^{t} B_{2}(s) e^{\theta_{13}(s)}\left[i_{1}(s)-i_{2}(s)\right] \\
& =-\int_{-2 \tau_{1}}^{-\tau_{1}} A\left(t+s+\tau_{1}\right) \\
& \cdot e^{\theta_{11}\left(t+s+\tau_{1}\right)}\left[x_{1}(t+s)-x_{2}(t+s)\right] d s \\
& -\int_{-\tau_{1}}^{0} B_{1}(t+s) e^{\theta_{12}(t+s)}\left[s_{1}(t+s)-s_{2}(t+s)\right] d s \\
& -\int_{-\tau_{1}}^{0} B_{2}(t+s) e^{\theta_{13}(t+s)}\left[i_{1}(t+s)-i_{2}(t+s)\right] d s
\end{aligned}
$$

which implies that

$$
\begin{aligned}
& \left|\int_{t-\tau_{1}}^{t}\left[x_{1}^{\prime}(s)-x_{2}^{\prime}(s)\right] d s\right| \\
& \leq A^{u} u^{*} \int_{-2 \tau_{1}}^{-\tau_{1}}\left|x_{1}(t+s)-x_{2}(t+s)\right| d s \\
& \quad+B_{1}^{u} v^{*} \int_{-\tau_{1}}^{0}\left|s_{1}(t+s)-s_{2}(t+s)\right| d s \\
& \quad+B_{2}^{u} w^{*} \int_{-\tau_{1}}^{0}\left|i_{1}(t+s)-i_{2}(t+s)\right| d s .
\end{aligned}
$$

Thus,

$$
\begin{aligned}
\left(x_{1}^{\prime}(t)\right. & \left.-x_{2}^{\prime}(t)\right) \operatorname{sgn}\left(x_{1}(t)-x_{2}(t)\right) \\
\leq & -A^{l} u_{*}\left|x_{1}(t)-x_{2}(t)\right| \\
& +\left(A^{u} u^{*}\right)^{2} \int_{-2 \tau_{1}}^{-\tau_{1}}\left|x_{1}(t+s)-x_{2}(t+s)\right| d s \\
& +A^{u} B_{1}^{u} u^{*} v^{*} \int_{-\tau_{1}}^{0}\left|s_{1}(t+s)-s_{2}(t+s)\right| d s \\
& +A^{u} B_{2}^{u} u^{*} w^{*} \int_{-\tau_{1}}^{0}\left|i_{1}(t+s)-i_{2}(t+s)\right| d s \\
& +B_{1}^{u} v^{*}\left|s_{1}(t)-s_{2}(t)\right|+B_{2}^{u} w^{*}\left|i_{1}(t)-i_{2}(t)\right| .
\end{aligned}
$$


Similarly, we can get

$$
\begin{aligned}
& \left|\int_{t-\tau_{1}}^{t}\left[s_{1}^{\prime}(s)-s_{2}^{\prime}(s)\right] d s\right| \\
& \leq \frac{C^{u} u^{*} v^{*}}{v_{*}} \int_{-2 \tau_{2}}^{-\tau_{2}}\left|x_{1}(t+s)-x_{2}(t+s)\right| d s \\
& \quad+\frac{C^{u} u^{*} v^{*}}{v_{*}} \int_{-2 \tau_{2}}^{0}\left|s_{1}(t+s)-s_{2}(t+s)\right| d s \\
& \quad+D_{11}^{u} v^{*} \int_{-\tau_{2}}^{0}\left|s_{1}(t+s)-s_{2}(t+s)\right| d s \\
& \quad+D_{12}^{u} w^{*} \int_{-\tau_{2}}^{0}\left|i_{1}(t+s)-i_{2}(t+s)\right| d s \\
& \quad+E_{1}^{u} w^{*} \int_{-\tau_{2}-\tau_{3}}^{-\tau_{3}}\left|i_{1}(t+s)-i_{2}(t+s)\right| d s,
\end{aligned}
$$

$$
\left(s_{1}^{\prime}(t)-s_{2}^{\prime}(t)\right) \operatorname{sgn}\left(s_{1}(t)-s_{2}(t)\right)
$$$$
\leq-D_{11}^{l} v_{*}\left|s_{1}(t)-s_{2}(t)\right|
$$$$
+\left(\frac{C^{u} u^{*} v^{*}}{v_{*}}\right)^{2} \int_{-2 \tau_{2}}^{-\tau_{2}}\left|x_{1}(t+s)-x_{2}(t+s)\right| d s
$$$$
+\frac{C^{u} u^{*} v^{*}}{v_{*}}\left|x_{1}\left(t-\tau_{2}\right)-x_{2}\left(t-\tau_{2}\right)\right|
$$$$
+\left(\frac{C^{u} u^{*} v^{*}}{v_{*}}\right)^{2} \int_{-2 \tau_{2}}^{0}\left|s_{1}(t+s)-s_{2}(t+s)\right| d s
$$

$$
+\frac{C^{u} D_{11}^{u} u^{*}\left(v^{*}\right)^{2}}{v_{*}} \int_{-\tau_{2}}^{0}\left|s_{1}(t+s)-s_{2}(t+s)\right| d s
$$$$
+\frac{C^{u} D_{12}^{u} u^{*} v^{*} w^{*}}{v_{*}} \int_{-\tau_{2}}^{0}\left|i_{1}(t+s)-i_{2}(t+s)\right| d s
$$$$
+\frac{C^{u} E_{1}^{u} u^{*} v^{*} w^{*}}{v_{*}} \int_{-\tau_{2}-\tau_{3}}^{-\tau_{3}}\left|i_{1}(t+s)-i_{2}(t+s)\right| d s
$$$$
+D_{12}^{u} w^{*}\left|i_{1}(t)-i_{2}(t)\right|
$$$$
+E_{1}^{u} w^{*}\left|i_{1}\left(t-\tau_{3}\right)-i_{2}\left(t-\tau_{3}\right)\right|,
$$

$$
\left|\int_{t-\tau_{1}}^{t}\left[i_{1}^{\prime}(s)-i_{2}^{\prime}(s)\right] d s\right|
$$$$
\leq \frac{E_{2}^{u} v^{*} w^{*}}{w_{*}} \int_{-\tau_{3}}^{0}\left|s_{1}(t+s)-s_{2}(t+s)\right| d s
$$$$
+\frac{E_{2}^{u} v^{*} w^{*}}{w_{*}} \int_{-2 \tau_{3}}^{0}\left|i_{1}(t+s)-i_{2}(t+s)\right| d s
$$$$
+D_{21}^{u} v^{*} \int_{-\tau_{3}}^{0}\left|s_{1}(t+s)-s_{2}(t+s)\right| d s
$$$$
+D_{22}^{u} w^{*} \int_{-\tau_{3}}^{0}\left|i_{1}(t+s)-i_{2}(t+s)\right| d s,
$$

$$
\begin{aligned}
\left(i_{1}^{\prime}(t)\right. & \left.-i_{2}^{\prime}(t)\right) \operatorname{sgn}\left(s_{1}(t)-s_{2}(t)\right) \\
\leq & -D_{22}^{l} w_{*}\left|i_{1}(t)-i_{2}(t)\right| \\
& +\left(\frac{E_{2}^{u} v^{*} w^{*}}{w_{*}}+D_{21}^{u} v^{*}\right)\left|s_{1}(t)-s_{2}(t)\right| \\
& +\left(\frac{E_{2}^{u} v^{*} w^{*}}{w_{*}}\right)^{2} \int_{-\tau_{3}}^{0}\left|s_{1}(t+s)-s_{2}(t+s)\right| d s \\
& +\left(\frac{E_{2}^{u} v^{*} w^{*}}{w_{*}}\right)^{2} \int_{-2 \tau_{3}}^{0}\left|i_{1}(t+s)-i_{2}(t+s)\right| d s \\
& +\frac{D_{21}^{u} E_{2}^{u}\left(v^{*}\right)^{2} w^{*}}{w_{*}} \int_{-\tau_{3}}^{0}\left|s_{1}(t+s)-s_{2}(t+s)\right| d s \\
& +\frac{D_{22}^{u} E_{2}^{u} v^{*}\left(w^{*}\right)^{2}}{w_{*}} \int_{-\tau_{3}}^{0}\left|i_{1}(t+s)-i_{2}(t+s)\right| d s .
\end{aligned}
$$

\section{Therefore,}

$$
\begin{aligned}
& D^{+} V_{1}(t) \leq-\lambda_{1} A^{l} u_{*}\left|x_{1}(t)-x_{2}(t)\right| \\
& -\left[\lambda_{2} D_{11}^{l} v_{*}-\lambda_{1} B_{1}^{u} v^{*}-\lambda_{3}\left(\frac{E_{2}^{u} v^{*} w^{*}}{w_{*}}+D_{21}^{u} v^{*}\right)\right] \\
& \times\left|s_{1}(t)-s_{2}(t)\right| \\
& -\left[\lambda_{3} D_{22}^{l} w_{*}-\lambda_{1} B_{2}^{u} w^{*}-\lambda_{2} D_{12}^{u} w^{*}\right]\left|i_{1}(t)-i_{2}(t)\right| \\
& +\lambda_{1}\left(A^{u} u^{*}\right)^{2} \int_{-2 \tau_{1}}^{-\tau_{1}}\left|x_{1}(t+s)-x_{2}(t+s)\right| d s \\
& +\lambda_{2}\left(\frac{C^{u} u^{*} v^{*}}{v_{*}}\right)^{2} \int_{-2 \tau_{2}}^{-\tau_{2}}\left|x_{1}(t+s)-x_{2}(t+s)\right| d s \\
& +\lambda_{1} A^{u} B_{1}^{u} u^{*} v^{*} \int_{-\tau_{1}}^{0}\left|s_{1}(t+s)-s_{2}(t+s)\right| d s \\
& +\lambda_{2} \frac{C^{u} D_{11}^{u} u^{*}\left(v^{*}\right)^{2}}{v_{*}} \int_{-\tau_{2}}^{0}\left|s_{1}(t+s)-s_{2}(t+s)\right| d s \\
& +\lambda_{2}\left(\frac{C^{u} u^{*} v^{*}}{v_{*}}\right)^{2} \int_{-2 \tau_{2}}^{0}\left|s_{1}(t+s)-s_{2}(t+s)\right| d s \\
& +\lambda_{3}\left(\frac{E_{2}^{u} v^{*} w^{*}}{w_{*}}\right)^{2} \int_{-\tau_{3}}^{0}\left|s_{1}(t+s)-s_{2}(t+s)\right| d s \\
& +\lambda_{3} \frac{D_{21}^{u} E_{2}^{u}\left(v^{*}\right)^{2} w^{*}}{w_{*}} \int_{-\tau_{3}}^{0}\left|s_{1}(t+s)-s_{2}(t+s)\right| d s \\
& +\lambda_{1} A^{u} B_{2}^{u} u^{*} w^{*} \int_{-\tau_{1}}^{0}\left|i_{1}(t+s)-i_{2}(t+s)\right| d s \\
& +\lambda_{2} \frac{C^{u} D_{12}^{u} u^{*} v^{*} w^{*}}{v_{*}} \int_{-\tau_{2}}^{0}\left|i_{1}(t+s)-i_{2}(t+s)\right| d s \\
& +\lambda_{2} \frac{C^{u} E_{1}^{u} u^{*} v^{*} w^{*}}{v_{*}} \int_{-\tau_{2}-\tau_{3}}^{-\tau_{3}}\left|i_{1}(t+s)-i_{2}(t+s)\right| d s
\end{aligned}
$$




$$
\begin{aligned}
& +\lambda_{3}\left(\frac{E_{2}^{u} v^{*} w^{*}}{w_{*}}\right)^{2} \int_{-2 \tau_{3}}^{0}\left|i_{1}(t+s)-i_{2}(t+s)\right| d s \\
& +\lambda_{3} \frac{D_{22}^{u} E_{2}^{u} v^{*}\left(w^{*}\right)^{2}}{w_{*}} \int_{-\tau_{3}}^{0}\left|i_{1}(t+s)-i_{2}(t+s)\right| d s \\
& +\lambda_{2} \frac{C^{u} u^{*} v^{*}}{v_{*}}\left|x_{1}\left(t-\tau_{2}\right)-x_{2}\left(t-\tau_{2}\right)\right| \\
& +\lambda_{2} E_{1}^{u} w^{*}\left|i_{1}\left(t-\tau_{3}\right)-i_{2}\left(t-\tau_{3}\right)\right| .
\end{aligned}
$$

On the other hand, according to the structure of $V_{i}(t), i=$ $2,3,4,5$, we can easily calculate the right derivatives $D^{+} V_{2}(t)$, $D^{+} V_{3}(t), D^{+} V_{4}(t)$, and $D^{+} V_{5}(t)$ along the solution of system (60) as follows:

$$
\begin{aligned}
& D^{+} V_{2}(t)=\left[\lambda_{1} \tau_{1}\left(A^{u} u^{*}\right)^{2}+\lambda_{2} \tau_{2}\left(\frac{C^{u} u^{*} v^{*}}{v_{*}}\right)^{2}\right] \\
& \cdot\left|x_{1}(t)-x_{2}(t)\right|-\lambda_{2}\left(\frac{C^{u} u^{*} v^{*}}{v_{*}}\right)^{2} \\
& \cdot \int_{-2 \tau_{2}}^{-\tau_{2}}\left|x_{1}(t+s)-x_{2}(t+s)\right| d s-\lambda_{1}\left(A^{u} u^{*}\right)^{2} \\
& \int_{-2 \tau_{1}}^{-\tau_{1}}\left|x_{1}(t+s)-x_{2}(t+s)\right| d s \\
& D^{+} V_{3}(t)=\left[\lambda_{1} \tau_{1} A^{u} B_{1}^{u} u^{*} v^{*}+2 \lambda_{2} \tau_{2}\left(\frac{C^{u} u^{*} v^{*}}{v_{*}}\right)^{2}\right. \\
& +\lambda_{2} \tau_{2} \frac{C^{u} D_{11}^{u} u^{*}\left(v^{*}\right)^{2}}{v_{*}}+\lambda_{3} \tau_{3}\left(\frac{E_{2}^{u} v^{*} w^{*}}{w_{*}}\right)^{2} \\
& \left.+\lambda_{3} \tau_{3} \frac{D_{21}^{u} E_{2}^{u}\left(v^{*}\right)^{2} w^{*}}{w_{*}}\right]\left|s_{1}(t)-s_{2}(t)\right| \\
& -\lambda_{1} A^{u} B_{1}^{u} u^{*} v^{*} \int_{-\tau_{1}}^{0}\left|s_{1}(t+s)-s_{2}(t+s)\right| d s \\
& -\lambda_{2}\left(\frac{C^{u} u^{*} v^{*}}{v_{*}}\right)^{2} \int_{-2 \tau_{2}}^{0}\left|s_{1}(t+s)-s_{2}(t+s)\right| d s \\
& -\lambda_{2} \frac{C^{u} D_{11}^{u} u^{*}\left(v^{*}\right)^{2}}{v_{*}} \int_{-\tau_{2}}^{0}\left|s_{1}(t+s)-s_{2}(t+s)\right| d s \\
& -\lambda_{3}\left(\frac{E_{2}^{u} v^{*} w^{*}}{w_{*}}\right)^{2} \int_{-\tau_{3}}^{0}\left|s_{1}(t+s)-s_{2}(t+s)\right| d s \\
& -\lambda_{3} \frac{D_{21}^{u} E_{2}^{u}\left(v^{*}\right)^{2} w^{*}}{w_{*}} \int_{-\tau_{3}}^{0}\left|s_{1}(t+s)-s_{2}(t+s)\right| d s ; \\
& D^{+} V_{4}(t)=\left[\lambda_{1} \tau_{1} A^{u} B_{2}^{u} u^{*} w^{*}+\lambda_{2} \tau_{2} \frac{C^{u} D_{12}^{u} u^{*} v^{*} w^{*}}{v_{*}}\right. \\
& +\lambda_{2} \tau_{2} \frac{C^{u} E_{1}^{u} u^{*} v^{*} w^{*}}{v_{*}}+2 \lambda_{3} \tau_{3}\left(\frac{E_{2}^{u} v^{*} w^{*}}{w_{*}}\right)^{2}
\end{aligned}
$$

$$
\begin{aligned}
& \left.+\lambda_{3} \tau_{3} \frac{D_{22}^{u} E_{2}^{u}\left(w^{*}\right)^{2} v^{*}}{w_{*}}\right]\left|i_{1}(t)-i_{2}(t)\right| \\
& -\lambda_{1} A^{u} B_{2}^{u} u^{*} w^{*} \int_{-\tau_{1}}^{0}\left|i_{1}(t+s)-i_{2}(t+s)\right| d s \\
& -\lambda_{2} \frac{C^{u} D_{12}^{u} u^{*} v^{*} w^{*}}{v_{*}} \int_{-\tau_{2}}^{0}\left|i_{1}(t+s)-i_{2}(t+s)\right| d s \\
& -\lambda_{2} \frac{C^{u} E_{1}^{u} u^{*} v^{*} w^{*}}{v_{*}} \int_{-\tau_{2}-\tau_{3}}^{-\tau_{3}}\left|i_{1}(t+s)-i_{2}(t+s)\right| d s \\
& -\lambda_{3}\left(\frac{E_{2}^{u} v^{*} w^{*}}{w_{*}}\right)^{2} \int_{-2 \tau_{3}}^{0}\left|i_{1}(t+s)-i_{2}(t+s)\right| d s \\
& -\lambda_{3} \frac{D_{22}^{u} E_{2}^{u}\left(w^{*}\right)^{2} v^{*}}{w_{*}} \int_{-\tau_{3}}^{0}\left|i_{1}(t+s)-i_{2}(t+s)\right| d s \\
& D^{+} V_{5}(t)=\frac{\lambda_{2} C^{u} u^{*} v^{*}}{v_{*}}\left|x_{1}(t)-x_{2}(t)\right| \\
& +\frac{\lambda_{2} C^{u} u^{*} v^{*}}{v_{*}}\left|x_{1}\left(t-\tau_{2}\right)-x_{2}\left(t-\tau_{2}\right)\right| \\
& +\lambda_{2} E_{1}^{u} w^{*}\left|i_{1}(t)-i_{2}(t)\right|-\lambda_{2} E_{1}^{u} w^{*} \mid i_{1}\left(t-\tau_{3}\right) \\
& -i_{2}\left(t-\tau_{3}\right) \mid \cdot
\end{aligned}
$$

From (78)-(79), by a simple reduction we can easily give the following estimations for the right derivatives of $V(t)$ :

$$
\begin{aligned}
& D^{+} V(t) \leq-\left[\lambda_{1} A^{l} u_{*}-\lambda_{1} \tau_{1}\left(A^{u} u^{*}\right)^{2}\right. \\
& \left.-\lambda_{2} \tau_{2}\left(\frac{C^{u} u^{*} v^{*}}{v_{*}}\right)^{2}-\frac{\lambda_{2} C^{u} u^{*} v^{*}}{v_{*}}\right]\left|x_{1}(t)-x_{2}(t)\right| \\
& -\left[\lambda_{2} D_{11}^{l} v_{*}-\lambda_{1} B_{1}^{u} v^{*}-\lambda_{3}\left(\frac{E_{2}^{u} v^{*} w^{*}}{w_{*}}+D_{21}^{u} v^{*}\right)\right. \\
& -\lambda_{1} \tau_{1} A^{u} B_{1}^{u} u^{*} v^{*}-2 \lambda_{2} \tau_{2}\left(\frac{C^{u} u^{*} v^{*}}{v_{*}}\right)^{2} \\
& -\lambda_{2} \tau_{2} \frac{C^{u} D_{11}^{u} u^{*}\left(v^{*}\right)^{2}}{v_{*}}-\lambda_{3} \tau_{3}\left(\frac{E_{2}^{u} v^{*} w^{*}}{w_{*}}\right)^{2} \\
& \left.-\lambda_{3} \tau_{3} \frac{D_{21}^{u} E_{2}^{u}\left(v^{*}\right)^{2} w^{*}}{w_{*}}\right]\left|s_{1}(t)-s_{2}(t)\right| \\
& \left.-\lambda_{3} \tau_{3} \frac{D_{22}^{u} E_{2}^{u}\left(w^{*}\right)^{2} v^{*}}{w_{*}}\right]\left|i_{1}(t)-i_{2}(t)\right| \\
& -\left[\lambda_{3} D_{22}^{l} w_{*}-\lambda_{1} B_{2}^{u} w^{*}-\lambda_{2} D_{12}^{u} w^{*}-\lambda_{2} E_{1}^{u} w^{*}\right. \\
& -\lambda_{1} \tau_{1} A^{u} B_{2}^{u} u^{*} w^{*}-\lambda_{2} \tau_{2} \frac{C^{u} D_{12}^{u} u^{*} v^{*} w^{*}}{v_{*}^{u} u^{*} v^{*} w^{*}}-2 \lambda_{3} \tau_{3}\left(\frac{E_{2}^{u} v^{*} w^{*}}{w_{*}}\right)^{2} \\
& -
\end{aligned}
$$


According to the definitions of $Q_{i j}(i, j=1,2,3)$, it follows from (80) and (C7) that

$$
\begin{aligned}
& D^{+} V(t) \\
& \quad \leq-\left(\left|x_{1}(t)-x_{2}(t)\right|\left|s_{1}(t)-s_{2}(t)\right|\left|i_{1}(t)-i_{2}(t)\right|\right) \\
& \quad\left(\begin{array}{lll}
Q_{11} & Q_{12} & Q_{13} \\
Q_{21} & Q_{22} & Q_{23} \\
Q_{31} & Q_{32} & Q_{33}
\end{array}\right)\left(\begin{array}{l}
\lambda_{1} \\
\lambda_{2} \\
\lambda_{3}
\end{array}\right)<0 .
\end{aligned}
$$

Thus, there exists a positive constant $\gamma>0$ such that

$$
D^{+} V(t) \leq-\gamma V(t), \quad t \in R^{+} .
$$

This means that the last condition of Lemma 3 is satisfied. And by Lemma 3, system (59) admits a unique uniformly asymptotically stable almost periodic solution $\left(x_{1}(t), s_{1}(t), i_{1}(t)\right)^{T}$.

Thus, by the transformation of (58), we can conclude that system (17) admits a unique uniformly asymptotically stable almost periodic solution $(u(t), v(t), w(t))^{T}=$ $\left(e^{x_{1}(t)}, e^{s_{1}(t)}, e^{i_{1}(t)}\right)^{T}$.

Finally, we will explain that system (3) has a unique uniformly asymptotically stable almost periodic solution.

In fact, from Lemmas 5 and 6, we know that

$$
\begin{gathered}
(X(t), S(t), I(t))^{T}=\left(\prod_{0<t_{k}<t}\left(1+h_{1 k}\right) u(t),\right. \\
\left.\prod_{0<t_{k}<t}\left(1+h_{2 k}\right) v(t), \prod_{0<t_{k}<t}\left(1+h_{3 k}\right) w(t)\right)^{T}
\end{gathered}
$$

is a solution of system (3). Since condition (C2) holds, similar to the proofs of Lemma 31 and Theorem 79 in [26], we can prove that $(X(t), S(t), I(t))^{T}$ is almost periodic. Therefore, $(X(t), S(t), I(t))^{T}$ is the unique uniformly asymptotically stable almost periodic solution of system (3) because of the uniqueness and the uniformly asymptotical stability of $(u(t), v(t), w(t))^{T}$.

This completes the proof of this theorem.

\section{Discussions and Conclusions}

As we know, mathematical modeling and mathematical analysis are an important means of understanding and predicting many kinds of phenomena in the nature. And an important application of the model proposed in this paper is to study the biological control strategy in the island ecosystems. Research shows that invasive species are a leading threat to biodiversity. Together with habitat degradation and humandriven atmospheric and oceanic alterations, biotic invasions are seen as major agents of global change. In face of invaders, native species may be put at danger and are frequently driven to extinction, as they are not likely to have evolved defences against mainland predators and grazers accidentally or deliberately introduced. For example, it is reported that five cats introduced to Marion Island in 1949 resulted in a population of more than 2,000 cats 25 years later, depleting nearly half a million burrowing petrel per year. They even caused the extinction of the Common Diving Petrel and severely affected some species of hole-nesting petrels. As a result, many scholars tried every way to control the population of the cats and keep the island's ecological balance until they introduced Feline Immunodeficiency Virus (FIV) to the cats (see [40]). FIV is a virus like AIDS in human; it is highly host-specific and has a low virulence; it persists for a long time before killing its host, allowing for multiple virus transmissions during the host's lifetime. It spreads mainly among the cats with strong physique and high birth rate directly. The virus breaks out in the body of the cat gradually for a period of time; then the predation capacity of the cats will decrease as time goes on. Thus, the model we proposed in this paper is very suitable to describe the dynamical relation between the cats and the burrowing petrels when introducing FIV to the cats, and different predation capacity of the susceptible cats and the infected cats is also considered. Furthermore, time delay of the virus attack in the body is also involved in the model. In addition, we introduced both chemical and biological control strategies into the model.

On the other hand, in the real nature, due to the interference of various factors, such as seasonal effects of the weather, food supplies, and mating habits, the coefficients of most of the systems are approximate to certain periodic functions. However, with the uncertainty of the interferences, the coefficients of the systems are not strictly periodic. Therefore, almost periodicity is a more common phenomenon than strict periodicity. Basing on the above background and the previous model proposed in [21], the authors proposed an ecological epidemic system with impulsive effects and multiple time delays on the model. It is supposed that all of the coefficients of the system are almost periodic, as well. Therefore, the proposed model in this paper is more original and extensive for the study of some specific problems. For instance, the previous model in [21] with nonimpulsive effects, single time delay, and periodic functions is one of the special cases in this paper.

By making full use of differential mean-value theorem with multivariables, differential inequalities, integral inequalities, absolute inequalities, and other mathematical analysis skills, sufficient conditions of the permanence for the system, the existence, and the uniformly asymptotical stability of a unique almost periodic solutions of the system are obtained. Thus, the mathematical results in the paper are quite new, and it may have some application value and practical significance for the prediction and control strategy for corresponding ecoepidemic systems.

\section{Conflict of Interests}

The authors declare that they have no competing interests.

\section{Acknowledgments}

The authors would like to thank the anonymous referees and editors for their helpful suggestions and comments 
which led to improvement of the original paper. This work is supported by the National Natural Science Foundation of China (51349011 and 41372301), Scientific Research Fund of Sichuan Provincial Education Department (11ZB192 and 14ZB0115), the Preeminent Youth Talent Project of Southwest University of Science and Technology (13ZX9109), and the Doctorial Research Fund of Southwest University of Science and Technology.

\section{References}

[1] Y. N. Xiao and F. Van Den Bosch, "The dynamics of an ecoepidemic model with biological control," Ecological Modelling, vol. 168, no. 1-2, pp. 203-214, 2003.

[2] B. Mukhopadhyay and R. Bhattacharyya, "Role of predator switching in an eco-epidemiological model with disease in the prey," Ecological Modelling, vol. 220, no. 7, pp. 931-939, 2009.

[3] S. Chakraborty, S. Pal, and N. Bairagi, "Dynamics of a ratiodependent eco-epidemiological system with prey harvesting," Nonlinear Analysis: Real World Applications, vol. 11, no. 3, pp. 1862-1877, 2010.

[4] M. Haque, "A predator-prey model with disease in the predator species only," Nonlinear Analysis. Real World Applications, vol. 11, no. 4, pp. 2224-2236, 2010.

[5] J. J. Li and W. J. Gao, "Analysis of a prey-predator model with disease in prey," Applied Mathematics and Computation, vol. 217, no. 8, pp. 4024-4035, 2010.

[6] K. Das, K. Kundu, and J. Chattopadhyay, "A predator-prey mathematical model with both the populations affected by diseases," Ecological Complexity, vol. 8, no. 1, pp. 68-80, 2011.

[7] B. W. Kooi, G. A. K. V. Voorn, and K. P. Das, "Stabilization and complex dynamics in a predator-prey model with predator suffering from an infectious disease," Ecological Complexity, vol. 8, no. 1, pp. 113-122, 2011.

[8] W. T. Wang, H. L. Liu, Z. Z. Li, Z. Guo, and Y. Yang, "Invasion dynamics of epidemic with the Allee effect," BioSystems, vol. 105, no. 1, pp. 25-33, 2011.

[9] X. B. Gao, Q. H. Pan, M. F. He, and Y. Kang, "A predator-prey model with diseases in both prey and predator," Physica A, vol. 392, no. 23, pp. 5898-5906, 2013.

[10] S. Jana and T. K. Kar, "Modeling and analysis of a prey-predator system with disease in the prey," Chaos, Solitons \& Fractals, vol. 47, no. 1, pp. 42-53, 2013.

[11] S. K. Sasmal and J. Chattopadhyay, "An eco-epidemiological system with infected prey and predator subject to the weak Allee effect," Mathematical Biosciences, vol. 246, no. 2, pp. 260-271, 2013.

[12] B. Sahoo and S. Poria, "Disease control in a food chain model supplying alternative food," Applied Mathematical Modelling, vol. 37, no. 8, pp. 5653-5663, 2013.

[13] R. K. Upadhyay, S. N. Raw, P. Roy, and V. Rai, "Restoration and recovery of damaged eco-epidemiological systems: application to the Salton SEA, California, USA," Mathematical Biosciences, vol. 242, no. 2, pp. 172-187, 2013.

[14] R. K. Upadhyay and P. Roy, "Spread of a disease and its effect on population dynamics in an eco-epidemiological system," Communications in Nonlinear Science and Numerical Simulation, vol. 19, no. 12, pp. 4170-4184, 2014.

[15] Y. H. Yang, W. D. Li, and G. Wang, "Can we use disease to control biological invasion?-a theoretical research," Ecological Modelling, vol. 277, pp. 97-107, 2014.
[16] X. Y. Zhou and J. G. Cui, "Stability and Hopf bifurcation analysis of an eco-epidemiological model with delay," Journal of the Franklin Institute, vol. 347, no. 9, pp. 1654-1680, 2010.

[17] M. Haque, S. Sarwardi, S. Preston, and E. Venturino, "Effect of delay in a Lotka-Volterra type predator-prey model with a transmissible disease in the predator species," Mathematical Biosciences, vol. 234, no. 1, pp. 47-57, 2011.

[18] R. Xu, "Mathematical analysis of the global dynamics of an ecoepidemiological model with time delay," Journal of the Franklin Institute, vol. 350, no. 10, pp. 3342-3364, 2013.

[19] R. Xu and S. Zhang, "Modelling and analysis of a delayed predator-prey model with disease in the predator," Applied Mathematics and Computation, vol. 224, pp. 372-386, 2013.

[20] X. Y. Shi, J. A. Cui, and X. Y. Zhou, "Stability and Hopf bifurcation analysis of an eco-epidemic model with a stage structure," Nonlinear Analysis. Theory, Methods \& Applications, vol. 74, no. 4, pp. 1088-1106, 2011.

[21] B. D. Tian, Y. H. Qiu, and N. Chen, "Periodic and almost periodic solution for a non-autonomous epidemic predatorprey system with time-delay," Applied Mathematics and Computation, vol. 215, no. 2, pp. 779-790, 2009.

[22] Y. Kuang, Delay Differential Equations with Applications in Population Dynamics, Academic Press, San Diego, Calif, USA, 1993.

[23] Z. X. Zheng, Theory of Functional Differential Equations, Anhui Education Press, 1994.

[24] V. Lakshmikantham, D. D. Bainov, and P. S. Simeonov, Theory of Impulsive Differential Equations, World Scientific, Singapore, 1989.

[25] D. D. Bainov and P. S. Simeonov, Impulsive Differential Eqations: Periodic Solutions and Applications, Burnt Mill, Longman Scientific and Technical, 1993.

[26] A. M. Samoilenko and N. A. Perestyuk, Impulsive Differential Equations, World Scientific, Singapore, 1995.

[27] H. Yu, S. Zhong, and R. P. Agarwal, "Mathematics analysis and chaos in an ecological model with an impulsive control strategy," Communications in Nonlinear Science and Numerical Simulation, vol. 16, no. 2, pp. 776-786, 2011.

[28] L. Nie, Z. Teng, L. Hu, and J. Peng, "Existence and stability of periodic solution of a predator-prey model with statedependent impulsive effects," Mathematics and Computers in Simulation, vol. 79, no. 7, pp. 2122-2134, 2009.

[29] R. Q. Shi, X. W. Jiang, and L. S. Chen, "The effect of impulsive vaccination on an SIR epidemic model," Applied Mathematics and Computation, vol. 212, no. 2, pp. 305-311, 2009.

[30] P. Georgescu and H. Zhang, "An impulsively controlled predator-pest model with disease in the pest," Nonlinear Analysis: Real World Applications, vol. 11, no. 1, pp. 270-287, 2010.

[31] L. Zou, Z. Xiong, and Z. Shu, "The dynamics of an ecoepidemic model with distributed time delay and impulsive control strategy," Journal of the Franklin Institute, vol. 348, no. 9, pp. 2332-2349, 2011.

[32] S. J. Gao, Y. Y. He, and L. S. Chen, "An epidemic model with pulses for pest management," Applied Mathematics and Computation, vol. 219, no. 9, pp. 4308-4321, 2013.

[33] B. Liu, Y. Wang, and B. L. Kang, "Dynamics on a pest management SI model with control strategies of different frequencies," Nonlinear Analysis: Hybrid Systems, vol. 12, pp. 66-78, 2014.

[34] Y. X. Xie, Z. H. Yuan, and L. J. Wang, "Dynamic analysis of pest control model with population dispersal in two patches and impulsive effect," Journal of Computational Science, vol. 5, no. 5, pp. 685-695, 2014. 
[35] Y. Wang, C.-D. Zheng, and E. Feng, "Stability analysis of mixed recurrent neural networks with time delay in the leakage term under impulsive perturbations," Neurocomputing, vol. 119, pp. 454-461, 2013.

[36] C. Wang, "Almost periodic solutions of impulsive BAM neural networks with variable delays on time scales," Communications in Nonlinear Science and Numerical Simulation, vol. 19, no. 8, pp. 2828-2842, 2014.

[37] T. W. Zhang, Y. K. Li, and Y. Ye, "On the existence and stability of a unique almost periodic solution of Schoener's competition model with pure-delays and impulsive effects," Communications in Nonlinear Science and Numerical Simulation, vol. 17, no. 3, pp. 1408-1422, 2012.

[38] Y. Nakata and Y. Muroya, "Permanence for nonautonomous Lotka-Volterra cooperative systems with delays," Nonlinear Analysis. Real World Applications, vol. 11, no. 1, pp. 528-534, 2010.

[39] C. Y. He, Almost Periodic Differential Equations, Higher Education Press, Beijing, China, 1992.

[40] N. M. Oliveira and F. M. Hilker, "Modelling disease introduction as biological control of invasive predators to preserve endangered prey," Bulletin of Mathematical Biology, vol. 72, no. 2, pp. 444-468, 2010. 


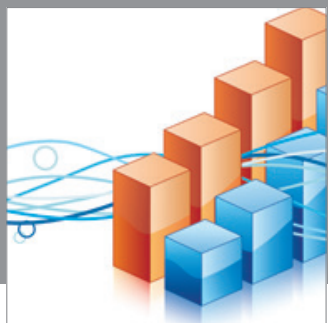

Advances in

Operations Research

mansans

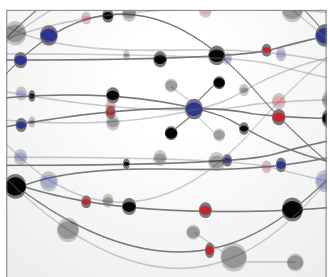

The Scientific World Journal
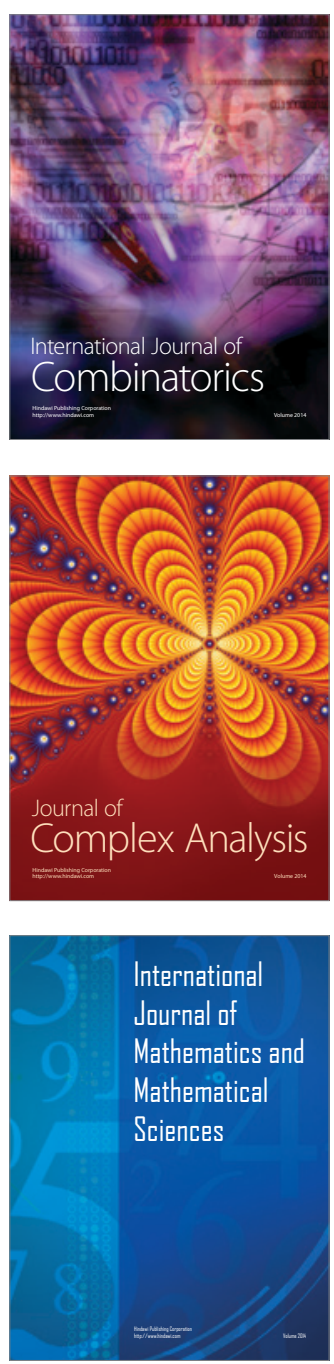
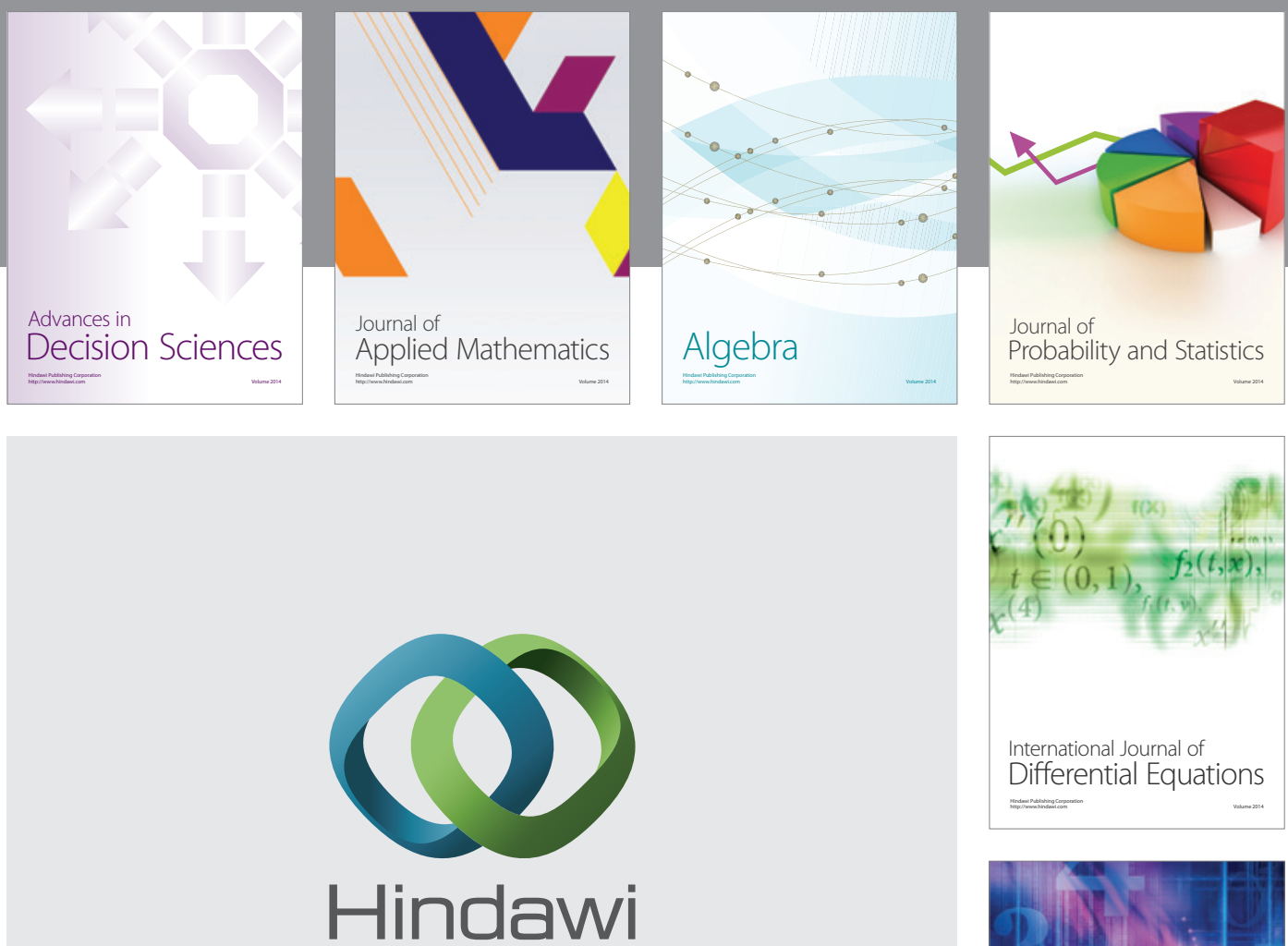

Submit your manuscripts at http://www.hindawi.com
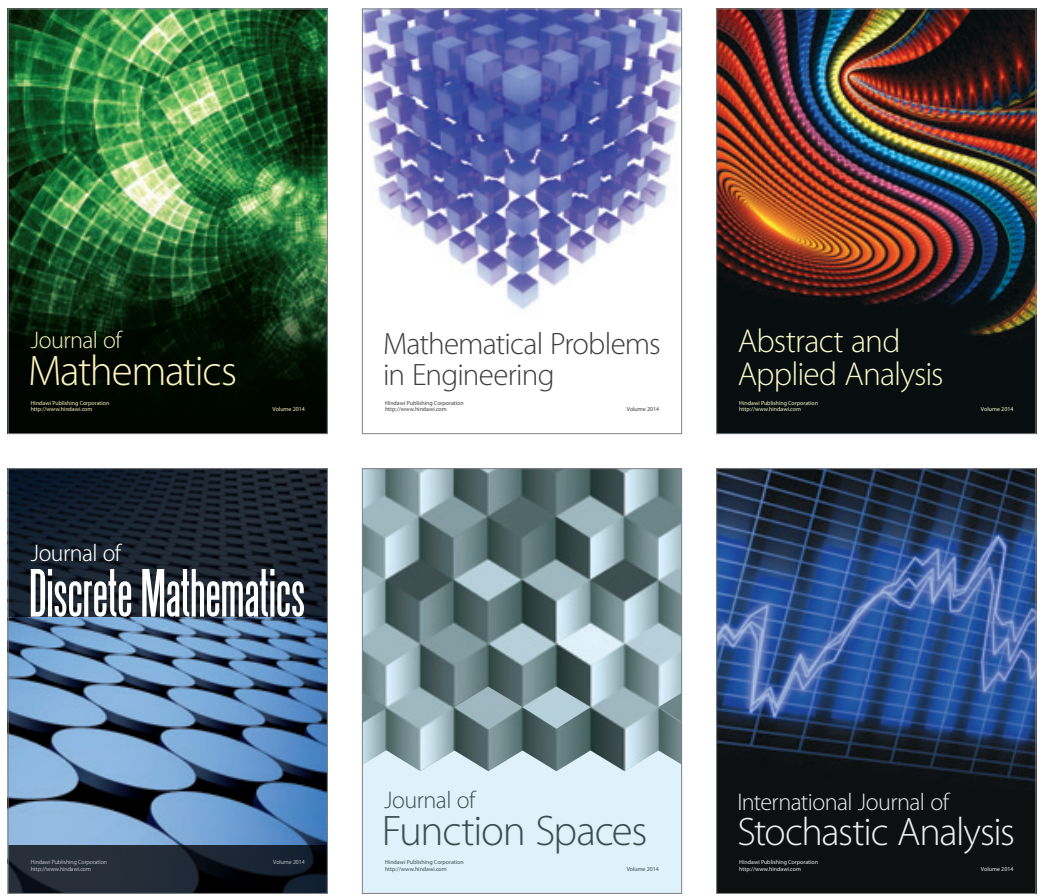

Journal of

Function Spaces

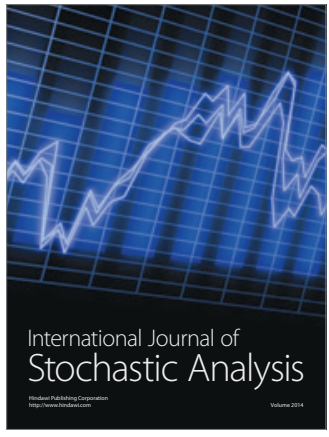

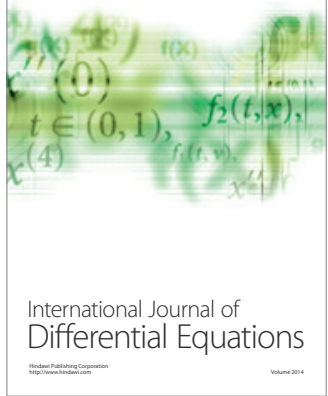
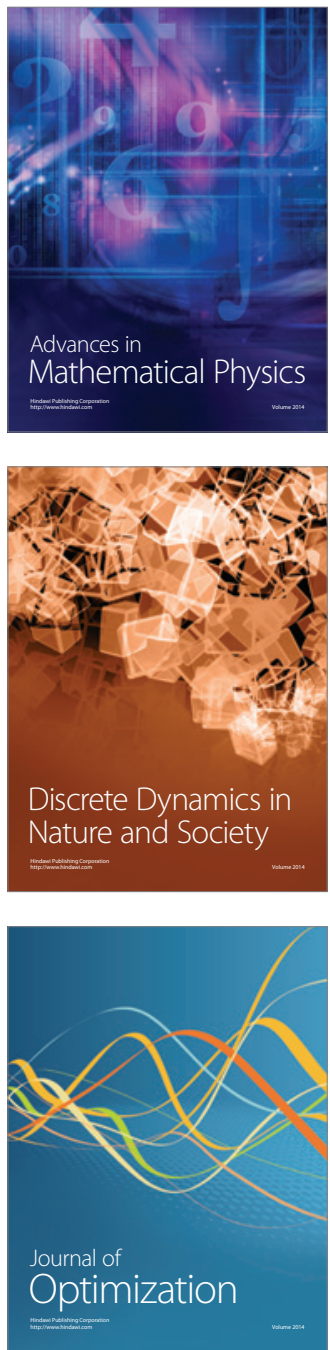